\title{
Propagation regimes of interfacial solitary waves in a three-layer fluid
}

\author{
O. E. Kurkina ${ }^{1}$, A. A. Kurkin ${ }^{1}$, E. A. Rouvinskaya ${ }^{1}$, and T. Soomere ${ }^{2,3}$ \\ ${ }^{1}$ Nizhny Novgorod State Technical University n.a. R. Alekseev, Nizhny Novgorod, Russia \\ ${ }^{2}$ Institute of Cybernetics, Tallinn University of Technology, Akadeemia tee 21, 12618 Tallinn, Estonia \\ ${ }^{3}$ Estonian Academy of Sciences, Kohtu 6, 10130 Tallinn, Estonia \\ Correspondence to: A. A. Kurkin (aakurkin@gmail.com)
}

Received: 5 December 2014 - Published in Nonlin. Processes Geophys. Discuss.: 6 January 2015

Revised: 22 January 2015 - Accepted: 23 January 2015 - Published: 4 March 2015

\begin{abstract}
Long weakly nonlinear finite-amplitude internal waves in a fluid consisting of three inviscid layers of arbitrary thickness and constant densities (stable configuration, Boussinesq approximation) bounded by a horizontal rigid bottom from below and by a rigid lid at the surface are described up to the second order of perturbation theory in small parameters of nonlinearity and dispersion. First, a pair of alternatives of appropriate KdV-type equations with the coefficients depending on the parameters of the fluid (layer positions and thickness, density jumps) are derived for the displacements of both modes of internal waves and for each interface between the layers. These equations are integrable for a very limited set of coefficients and do not allow for proper description of several near-critical cases when certain coefficients vanish. A more specific equation allowing for a variety of solitonic solutions and capable of resolving most near-critical situations is derived by means of the introduction of another small parameter that describes the properties of the medium and rescaling of the ratio of small parameters. This procedure leads to a pair of implicitly interrelated alternatives of Gardner equations (KdV-type equations with combined nonlinearity) for the two interfaces. We present a detailed analysis of the relationships for the solutions for the disturbances at both interfaces and various regimes of the appearance and propagation properties of soliton solutions to these equations depending on the combinations of the parameters of the fluid. It is shown that both the quadratic and the cubic nonlinear terms vanish for several realistic configurations of such a fluid.
\end{abstract}

\section{Introduction}

There are many important topics related to internal waves (IWs) in the ocean. Large IWs are highly significant for sediment resuspension and transport (Bogucki and Redekopp, 1999; Stastna and Lamb, 2008; Reeder et al., 2011) and for the biology on the continental shelf (Sandstrom and Elliott, 1984). The currents forced by large or breaking IWs cause powerful forces on marine platforms and submersibles. The associated strong distortion of the density field has a severe impact on acoustic signaling (Apel et al., 2007; Chin-Bing et al., 2009; Warn-Varnas et al., 2009; Sridevi et al., 2011). Their capacity to break and impact the local microstructure has major consequences for the understanding of interior ocean mixing (Muller and Briscoe, 2000). Internal waves are believed to be responsible for substantial damage (Osborne, 2010). High water velocities in intense IWs can create enormous local loads and bending moments and represent a potential danger to off-shore structures, such as oil platforms, drill rigs, etc. The danger from IWs is considered so critical that, similar to the systems of tsunami warning, the potential for automated detection systems for large-amplitude IWs (internal soliton early warning systems) is being discussed now. Such systems were even tested to support drilling campaigns and guarantee the safety of drilling platforms (Stober and Moum, 2011).

Most of the studies of IWs focus on large-amplitude localized IWs, which propagate in relatively shallow areas of oceans, shelf regions or semi-sheltered seas. A fascinating feature of many waves of this kind is that they propagate for a long time without any significant change in their en- 
ergy or shape as solitons do (Jeans, 1995). They can be interpreted and adequately described as internal solitary waves (ISWs) and are often referred to as internal solitons (Ostrovsky and Stepanyants, 1989; Grimshaw, 2001). Observations of large-amplitude ISWs are presented in many original research papers and reviews (Holloway et al., 2001; Jackson, 2004; Ostrovsky and Stepanyants, 2005; Sabinin and Serebryany, 2005; Vlasenko et al., 2005; Apel et al., 2007; Grimshaw et al., 2007).

The concept of layered fluid, widely used for both theoretical research and applications, is convenient for the description of IWs because wave motion in such environments is described by equations containing a small number of parameters and often allowing for analytical studies of the properties of solutions. These simple models are able to mirror the basic properties of the actual internal wave systems. The efficiency of the two-layer model - the simplest system basically representing the key properties of IWs - has been established in numerous analytical and numerical studies as well as by means of in situ observations and laboratory experiments (Funakoshi, 1985; Funakoshi and Oikawa, 1986; Mirie and Pennell, 1989; Choi and Camassa, 1996, 1999; Pullin and Grimshaw, 1998; Craig et al., 2004; Guyenne, 2006; Zahibo et al., 2007; Camassa et al., 2010).

The two-layer model only conditionally represents the vertical structure of seas and oceans. Its direct extension - threelayerstratification - has proved to be a proper approximation of the sea water density profile in some basins in the world ocean with specific hydrological conditions (Knauss, 1996; Leppäranta and Myrberg, 2009; Kurkina et al., 2011a). Three-layer models are more complex than two-layer systems, because of the increased number of set-up parameters, but they represent new dynamical effects and allow for much more analytical progress compared to the arbitrary stratified medium. Another advantage of three-layer models in contrast to two-layer ones is the ability to describe two modes of IWs, their properties and possible interactions.

Nonlinear theory of IWs in a three-layer fluid with constant density in each layer is presented in the literature in the framework of different approaches. Simplified equations for three-layer conjugate flows are derived in Lamb (2000) and Rusås and Grue (2002), and some analytic results are obtained to understand conjugate flows and, in consequence, large-amplitude, flat-centered (table-like) solitary internal waves of both modes. Comparison with solutions obtained for continuous stratifications is also given in Lamb (2000). A fully nonlinear numerical method for the calculation of solitary waves in a three-layer fluid (Rusås and Grue, 2002) allows for the investigation of both mode- 1 and mode2 waves, including broad flat-centered waves and extreme (overhanging) waves. The similarity of mode- 1 waves to the interfacial waves in a two-layer fluid is the probable reason why the analysis is mainly performed for situations when one of the layers is relatively thin. These models have greatly contributed to the understanding of nonlinear internal wave properties in three-layer fluids and flows. They are, however, often very complex, and the results are not easy to visualize. The drawback is that the analytical progress is limited and numerical computations should be involved to obtain final localized solutions and to analyze their properties.

Although contemporary numerical methods and fully nonlinear approaches such as the above-discussed method of conjugate flows allow for extensive studies into properties of highly nonlinear IWs, many specific features can still be recognized, analyzed and understood using classical methods for analytical studies into IWs in the weakly nonlinear framework. Such fully analytical methods make it possible to establish exactly the appearance of disturbances of different shapes and amplitudes, and, more importantly, to understand the specific features of the behavior of waves corresponding to the situation where a substantial change in the overall regime of wave propagation is possible (Kurkina et al., 2011a).

Weakly nonlinear analytical models of different levels for IWs in a three-layer fluid have been developed for some specific situations. The properties of mode-1 IWs in a symmetric three-layer fluid (when the undisturbed state is symmetric about the mid-depth) were analyzed up to the second order in nonlinearity by Grimshaw et al. (1997). An extension involving up to the fourth-order nonlinear terms was derived in Kurkina et al. (2011b). The model of Yang et al. (2010) involved both modes of IWs in a general three-layer ocean, the first order in nonlinearity and dispersion (Korteweg-de Vries (KdV) approximation; only mode-2 waves were analyzed). The weakly nonlinear theory for a continuously stratified fluid can be used to derive the parameters of evolution equations for IWs in a three-layer fluid (Grimshaw et al., 1997; Yang et al., 2010). These parameters for continuous stratification are given in the form of integral expressions including the vertical mode (whose shape is determined by the density stratification), its higher-order corrections and their derivatives (Lamb and Yan, 1996; Pelinovskii et al., 2000; Grimshaw et al., 2002). When this technique is adapted to a layered model (with a piecewise continuous density profile), the relevant integrands contain very complex expressions (especially for higher-order corrections and coefficients) involving generalized functions, and analytical progress in their analysis is not always possible. Therefore, it is preferable initially to use equations for layered models and to apply asymptotic expansions to them directly. This approach has been used, for instance, in Kurkina et al. (2011b) and Koop and Butler (1981).

A specific feature of weakly nonlinear equations for a three-layer fluid is that some coefficients at the nonlinear terms may vanish for certain modes and certain specific density stratifications (Kakutani and Yamasaki, 1978; Grimshaw et al., 1997; Yang et al., 2010). This feature is common for several wave classes in stratified environments (Soomere, 2003). Some coefficients at the nonlinear terms may vanish even in a two-layer fluid with the surface tension between the 

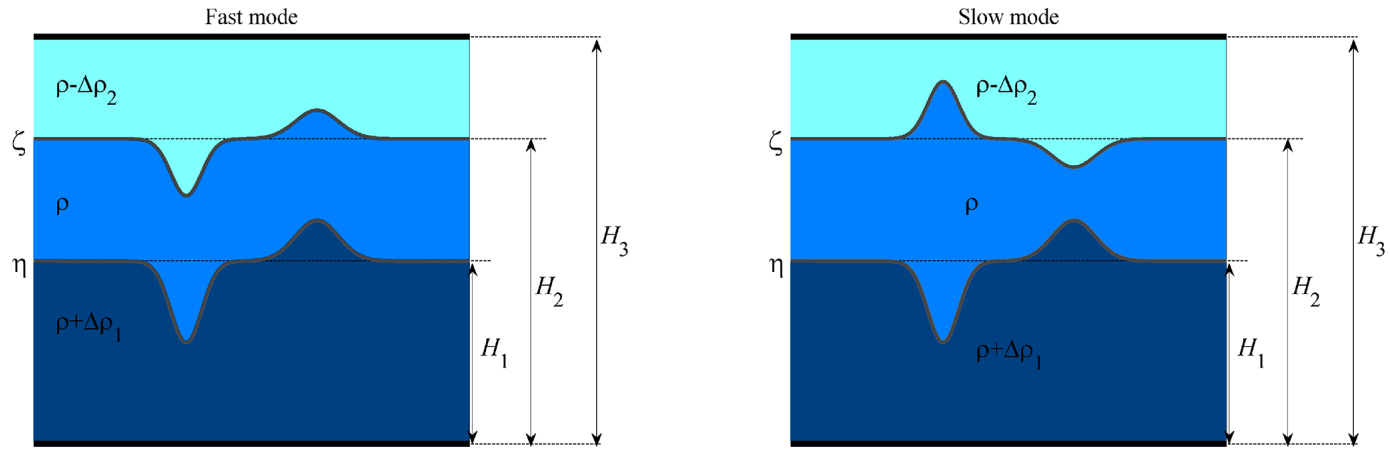

Figure 1. Definition sketch of the three-layer fluid and internal waves of mode-1 (left) and mode-2 (right).

layers (Giniyatullin et al., 2014; Kurkina et al., 2015). In such cases, it is often necessary to account for higher-order nonlinear terms to describe the motion adequately. In the case of IWs in a three-layer fluid, it is necessary to produce a secondorder weakly nonlinear theory to resolve some of such situations. The attempts in this direction have so far been limited to the specific case of symmetric stratification (Kurkina et al., 2011b).

We develop here an analytic model for long IWs of finite amplitude for the three-layer fluid with an arbitrary combination of the layers' thicknesses. Such a vertical structure much better matches the properties of real seas and oceans. The relevant nonlinear evolutionary equations are obtained first with the use of an asymptotic procedure from the governing equations for three homogeneous fluid layers. The derivation of these equations relies on the second order of the perturbation theory for both interfaces and for the waves of each mode. The developed framework makes it possible to investigate the properties of both modes of long internal gravity waves in a three-layer fluid. The key development is that, for each equation, the coefficients at the nonlinear terms and terms expressing linear and nonlinear dispersion of waves are expressed explicitly via parameters of the fluid configuration. This makes it possible to analyze comprehensively the behavior and signs of all coefficients. The necessary order of the equations is discussed and determined for each case. Special attention is paid to the situations when the nonlinear terms of the lowest order of the perturbation theory can vanish. For such situations, a particular rescaling is performed in order to balance the nonlinear and dispersion terms in the equations.

\section{Governing equations}

As usual in the analysis of long internal waves, let us consider a model situation of irrotational motions in a three-layer inviscid fluid with undisturbed interface positions $z=H_{1,2}$ $\left(H_{2} \geq H_{1}\right)$ and total thickness $H_{3}\left(H_{3} \geq H_{2}\right)$ overlying a flat horizontal bottom located at $z=0$ in the approximation of a rigid lid on the surface of the fluid (Fig. 1). The reference density of the middle layer is $\rho_{2}=\rho$ and the densities in the lowermost and uppermost layers are $\rho_{1}=\rho+\Delta \rho_{1}$ and $\rho_{3}=\rho-\Delta \rho_{2}$, respectively. We employ the Boussinesq approximation and assume that densities in the layers differ insignificantly $\left(\Delta \rho_{1,2} / \rho \ll 1\right)$. In this case, the equations of the motion are Laplace equations for the velocity potential $\Phi_{i}$ in each layer:

$\nabla^{2} \Phi_{i}=0, \quad i=1,2,3$

The kinematic boundary conditions at the bottom and at the fixed upper boundary reduce to the condition that vertical velocities at these boundaries vanish:

$\Phi_{1 z}=0$ at $z=0, \Phi_{3 z}=0$ at $z=H_{3}$.

Here and further on, the subscripts $x, y, z$ or $t$ denote a partial derivative along the respective coordinate $(x, y, z)$ or with respect to time $t$. The classical kinematic and dynamic boundary conditions at the interfaces between the layers are as follows (Kurkina et al., 2011b):

$$
\left.\begin{array}{l}
\eta_{t}+\Phi_{1_{x}} \eta_{x}-\Phi_{1_{z}}=0 \\
\eta_{t}+\Phi_{2_{x}} \eta_{x}-\Phi_{2_{z}}=0 \\
\rho_{1}\left(\Phi_{1_{t}}+\frac{1}{2}\left(\nabla \Phi_{1}\right)^{2}+g \eta\right) \\
=\rho_{2}\left(\Phi_{2_{t}}+\frac{1}{2}\left(\nabla \Phi_{2}\right)^{2}+g \eta\right)
\end{array}\right\} z=H_{1}+\eta(x, t),
$$

Here, the unknown functions $\eta(x, t)$ and $\zeta(x, t)$ denote the instantaneous position of the interface between the bottom and middle layers and between the upper and middle layers, respectively. 


\section{Asymptotic procedure}

The appearance of the proper evolution equation for a phenomenon in a realistic environment may substantially depend on the characteristic scales of the processes to be highlighted or analyzed. Similarly to Kurkina et al. (2011b), our goal is to derive an equation for small (but finite) amplitude longwave motions in a layered fluid. This goal naturally implies two small parameters. Firstly, the typical horizontal scale of wave motion $L$ (equivalently, the typical wave length for linear or nonlinear wave trains) considerably exceeds the fluid depth $H$. This assertion $L \gg H$ not only ensures that a small parameter $H / L$ is present in the system, but also matches the usual properties of environments supporting the IW motion in natural water bodies. It also fits the basic assumptions of common derivations of the $\mathrm{KdV}$ equation and its generalizations. Another small parameter naturally emerges from the restriction that the amplitude $a$ of the disturbances to the interfaces' positions from their undisturbed location is small compared to the fluid depth. This assertion gives rise to the parameter of nonlinearity $\varepsilon=a / H \ll 1$.

Our specific interest is to describe long-living solitary waves, for which the dispersion in some sense balances the impact of nonlinearity. As will be shown below, a convenient parameter that characterizes the role of dispersion in wave propagation is $\mu=H^{2} / L^{2}$. As the existence of long-living nonlinear wave motions and solitary waves usually presumes a specific balance between the terms representing nonlinear and dispersive effects, we assume that $\varepsilon \sim \mu^{0}$.

These assumptions and introduced parameters make it possible to perform the analysis of the problem Eqs. (1)-(4) in nondimensional form. The standard procedure of introducing nondimensional coordinates into Eqs. (1)-(4) under these assumptions leads to the following boundary problem:

$$
\begin{array}{ll}
\Phi_{1_{z z}}+\varepsilon \Phi_{1_{x x}}=0, & 0 \leq z \leq H_{1}, \\
\Phi_{2_{z z}}+\varepsilon \Phi_{2_{x x}}=0, & H_{1} \leq z \leq H_{2}, \\
\Phi_{3_{z z}}+\varepsilon \Phi_{3_{x x}}=0, & H_{2} \leq z \leq H_{3}, \\
\Phi_{1_{z}}=0 \text { at } z=0, & \Phi_{3_{z}}=0 \text { at } z=H_{3}
\end{array}
$$

$$
\left.\begin{array}{l}
\eta_{t}+\varepsilon \Phi_{1_{x}} \eta_{x}-\Phi_{1_{z}}=0, \\
\eta_{t}+\varepsilon \Phi_{2_{x}} \eta_{x}-\Phi_{2_{z}}=0 \\
\rho_{1}\left(\Phi_{1_{t}}+\frac{1}{2} \varepsilon\left(\Phi_{1_{x}}\right)^{2}+\frac{1}{2}\left(\Phi_{1_{z}}\right)^{2}\right. \\
+g \eta)=\rho_{2}\left(\Phi_{2_{t}}+\frac{1}{2} \varepsilon\left(\Phi_{2_{x}}\right)^{2}\right. \\
\left.+\frac{1}{2}\left(\Phi_{2_{z}}\right)^{2}+g \eta\right)
\end{array}\right\} z=H_{1}+\eta(x, t),
$$

$$
\left.\begin{array}{l}
\zeta_{t}+\varepsilon \Phi_{2_{x}} \zeta_{x}-\Phi_{2_{z}}=0 \\
\zeta_{t}+\varepsilon \Phi_{3_{x}} \zeta_{x}-\Phi_{3_{z}}=0 \\
\rho_{2}\left(\Phi_{2_{t}}+\frac{1}{2} \varepsilon\left(\Phi_{2_{x}}\right)^{2}+\frac{1}{2}\left(\Phi_{2_{z}}\right)^{2}\right. \\
+g \zeta)=\rho_{3}\left(\Phi_{3_{t}}+\frac{1}{2} \varepsilon\left(\Phi_{3_{x}}\right)^{2}\right. \\
\left.+\frac{1}{2}\left(\Phi_{3_{z}}\right)^{2}+g \zeta\right)
\end{array}\right\} z=H_{2}+\zeta(x, t)
$$

The asymptotic analysis of the resulting equations is straightforward. As the procedure is fairly cumbersome, largely follows a well-known approach (Koop and Butler, 1981) and provides almost no instructive aspects, we omit its details and present only its basic steps. First, all unknown functions are expanded into Taylor series in the vicinity of one of the interfaces:

$$
\begin{aligned}
& f\left(x, z=H_{1}+\eta(x, t), t\right)=\left.\sum_{j=0}^{\infty} \frac{\eta^{j}}{j !} \frac{\partial^{j} f}{\partial z^{j}}\right|_{z=H_{1}}, \\
& f\left(x, z=H_{2}+\zeta(x, t), t\right)=\left.\sum_{j=0}^{\infty} \frac{\zeta^{j}}{j !} \frac{\partial^{j} f}{\partial z^{j}}\right|_{z=H_{2}} .
\end{aligned}
$$

All constituents of the resulting series are then expanded into power series with respect to powers of the parameter $\varepsilon \ll 1$ :

$$
\begin{aligned}
& \eta=\varepsilon\left(\eta_{0}+\varepsilon \eta_{1}+\varepsilon^{2} \eta_{2}+\ldots\right), \\
& \zeta=\varepsilon\left(\zeta_{0}+\varepsilon \zeta_{1}+\varepsilon^{2} \zeta_{2}+\ldots\right) .
\end{aligned}
$$

It is easy to show that all the potentials $\Phi_{i} \sim \sqrt{\varepsilon}$; consequently, the power series for the potentials are as follows:

$\Phi_{i}=\sqrt{\varepsilon}\left(\varphi_{i, 0}+\varepsilon \varphi_{i, 1}+\varepsilon^{2} \varphi_{i, 2}+\ldots\right), \quad i=1,2,3$,

Finally, we employ the technique of multiple temporal and spatial scales (Engelbrecht et al., 1988; Nayfeh, 2000). The "slow" time and "stretched" reference frame coordinate are introduced as follows:

$\xi=\varepsilon^{1 / 2}(x-c t), \quad \tau=\varepsilon^{3 / 2} t$.

Here, $c$ denotes the (as yet unknown) phase speed of free linear internal waves. This substitution is equivalent to the following replacement of the operators of partial derivatives:

$\frac{\partial}{\partial x}=\varepsilon^{1 / 2} \frac{\partial}{\partial \xi}, \quad \frac{\partial}{\partial t}=-\varepsilon^{1 / 2} c \frac{\partial}{\partial \xi}+\varepsilon^{3 / 2} \frac{\partial}{\partial \tau}$.

Substitution of expansions (Eqs. 11-12) and definitions of modified coordinates (Eqs. 13-14) into Eqs. (5)-(10) leads to an infinite system of equations with respect to the elements of expansions (Eqs. 11-12). Equations of the resulting system 
are scaled by one of the multiples $\varepsilon^{1 / 2}$ or $\varepsilon^{3 / 2}$, where they arise. The obtained system can be solved recursively until any desired order. The system of equations obtained in the leading (lowest) order $\left(\sim \varepsilon^{0}\right)$ for the elements of expansions (Eqs. 11-13) has the form (Ruvinskaya et al., 2010)

$$
\begin{aligned}
& \varphi_{i, 0_{z z}}=0, \quad i=1,2,3, \\
& \varphi_{1,0_{z}}=0 \text { at } z=0, \quad \varphi_{3,0_{z}}=0 \text { at } z=H_{3},
\end{aligned}
$$

$$
\left.\begin{array}{l}
\varphi_{1,0_{z}}=0, \quad \varphi_{2,0_{z}}=0 \\
-(\rho+\Delta \rho) c \varphi_{1,0_{\xi}}+\Delta \rho g \eta_{0}=-\rho c \varphi_{2,0_{\xi}}
\end{array}\right\} z=H_{1},
$$

$$
\left.\begin{array}{l}
\varphi_{2,0_{z}}=0, \quad \varphi_{3,0_{z}}=0 \\
-\rho c \varphi_{2,0_{\xi}}+r \Delta \rho g \zeta_{0}=-(\rho-r \Delta \rho) c \varphi_{3,0_{\xi}}
\end{array}\right\} z=H_{2},
$$

where $\Delta \rho_{1}=\Delta \rho, \Delta \rho_{2}=r \Delta \rho$. It follows from Eqs. (15)(18) that the functions $\varphi_{i, 0}, i=1,2,3$ do not depend on the coordinate $z$. is

The system of equations obtained in the first order $\left(\sim \varepsilon^{1}\right)$

$\varphi_{i, 1_{z z}}+\varphi_{i, 0_{\xi \xi}}=0, \quad i=1,2,3$,

$\varphi_{1,1_{z}}(z=0)=0, \quad \varphi_{3,1_{z}}\left(z=H_{3}\right)=0$,

$$
\left.\begin{array}{l}
c \eta_{0_{\xi}}+\varphi_{1,1_{z}}=0, \quad c \eta_{0_{\xi}}+\varphi_{2,1_{z}}=0 \\
(\rho+\Delta \rho)\left(\varphi_{1,0_{\tau}}-c \varphi_{1,1_{\xi}}+\frac{1}{2} \varphi_{1,0_{\xi}}\right)+\Delta \rho g \eta_{1} \\
=\rho\left(\varphi_{2,0_{\tau}}-c \varphi_{2,1_{\xi}}+\frac{1}{2} \varphi_{2,0_{\xi}}^{2}\right)
\end{array}\right\} z=H_{1},
$$

$$
\left.\begin{array}{l}
c \zeta_{0_{\xi}}+\varphi_{2,1_{z}}=0, \quad c \zeta_{0_{\xi}}+\varphi_{3,1_{z}}=0 \\
\rho\left(\varphi_{2,0_{\tau}}-c \varphi_{2,1_{\xi}}+\frac{1}{2} \varphi_{2,0_{\xi}}^{2}\right)+\Delta \rho g \zeta_{1} \\
=(\rho-r \Delta \rho)\left(\varphi_{3,0_{\tau}}-c \varphi_{3,1_{\xi}}+\frac{1}{2} \varphi_{3,0_{\xi}}^{2}\right)
\end{array}\right\} z=H_{2} .
$$

From Eqs. (19)-(21), we obtain the following relationships:

$\varphi_{1,1_{z}}=-\varphi_{1,0_{\xi \xi}} z, \quad \varphi_{3,1_{z}}=\varphi_{3,0_{\xi \xi}}\left(H_{3}-z\right)$,

$\varphi_{1,0_{\xi \xi}} H_{1}=c \eta_{0_{\xi}}, \quad \varphi_{2,0_{\xi \xi}}\left(H_{1}-H_{2}\right)=c\left(\eta_{0}-\zeta_{0}\right)_{\xi}$,

$\varphi_{3,0_{\xi \xi}}\left(H_{2}-H_{3}\right)=c \zeta_{0_{\xi}}$.

Equations (22) and (23) together with Eqs. (17) and (18) allow one to define the phase speed $c$ of the linear IWs. The relevant equation is bi-quadratic and, not unexpectedly, reveals that two wave modes exist in this system. One of them results in synchronous in-phase movements of both the interfaces. The other mode is antisymmetric: the motions of the interfaces are synchronous but have opposite directions
(Fig. 1). The corresponding expressions for the phase speeds are

$$
\begin{aligned}
c^{ \pm 2} & =\frac{1}{2} \frac{\Delta \rho}{\rho} g H_{3}\left(l_{1}\left(1-l_{1}\right)+r l_{2}\left(1-l_{2}\right) \pm d\right) \\
& =\frac{1}{2} \frac{\Delta \rho}{\rho} g H_{3} \frac{1}{q^{ \pm}},
\end{aligned}
$$

where

$d=\sqrt{r^{2} l_{2}^{2}\left(1-l_{2}\right)^{2}-2 r l_{1}\left(1-l_{2}\right)\left(l_{2}-l_{1}\left(2-l_{2}\right)\right)+l_{1}^{2}\left(1-l_{1}\right)^{2}}$

and $l_{1,2}=H_{1,2} / H_{3}$. The symmetric mode of internal waves has the larger phase speed $c^{+}$, corresponding to the " + " sign before $d$ in Eq. (24). For this reason, we call it fast mode, or mode-1. The antisymmetric mode, whose phase speed is $c^{-}$, is called slow mode, or mode-2. Below we shall consider the nonlinear motions of both modes.

The first-order and second-order corrections to the linear solution satisfy the following equations:

$$
\begin{aligned}
& \varepsilon^{2}: \eta_{1_{\tau}}^{ \pm}+\alpha^{ \pm} \eta_{1}^{ \pm} \eta_{1_{\xi}}^{ \pm}+\beta^{ \pm} \eta_{1_{\xi \xi \xi}^{ \pm}}^{ \pm}=0, \\
& \zeta_{1_{\tau}}^{ \pm}+\widetilde{\alpha}^{ \pm} \zeta_{1}^{ \pm} \zeta_{1_{\xi}}^{ \pm}+\widetilde{\beta}^{ \pm} \zeta_{1_{\xi \xi \xi}}^{ \pm}=0,
\end{aligned}
$$

$$
\begin{aligned}
\varepsilon^{3}: & \eta_{2_{\tau}}^{ \pm}+\alpha^{ \pm}\left(\eta_{1}^{ \pm} \eta_{2}^{ \pm}\right)_{\xi}+\beta^{ \pm} \eta_{2_{\xi \xi \xi}}^{ \pm}+\alpha_{1}^{ \pm} \eta_{1}^{ \pm 2} \eta_{1_{\xi}}^{ \pm} \\
& +\beta_{1}^{ \pm} \eta_{1,5_{\xi}}^{ \pm}+\gamma_{1}^{ \pm} \eta_{1}^{ \pm} \eta_{1_{\xi \xi \xi}^{ \pm}}^{ \pm}+\gamma_{2}^{ \pm} \eta_{1_{\xi}}^{ \pm} \eta_{1_{\xi \xi}}^{ \pm}=0, \\
\zeta_{2_{\tau}}^{ \pm} & \widetilde{\alpha}^{ \pm}\left(\zeta_{1}^{ \pm} \zeta_{2}^{ \pm}\right)_{\xi}+\widetilde{\beta}^{ \pm} \zeta_{2_{\xi \xi \xi}}^{ \pm}+\widetilde{\alpha}_{1}^{ \pm} \zeta_{1}^{ \pm 2} \zeta_{1_{\xi}}^{ \pm}+\widetilde{\beta}_{1}^{ \pm} \zeta_{1,5_{\xi}}^{ \pm} \\
+ & \widetilde{\gamma}_{1}^{ \pm} \zeta_{1}^{ \pm} \zeta_{\xi_{\xi \xi \xi}}^{ \pm}+\widetilde{\gamma}_{2}^{ \pm} \zeta_{1_{\xi}}^{ \pm} \zeta_{l_{\xi \xi}}^{ \pm}=0 .
\end{aligned}
$$

The coefficients of Eqs. (25) and (26) are presented in the Appendix. The first-order equations (Eq. 25) are, as expected, the well-known $\mathrm{KdV}$ equations that describe the motions of both the interfaces. The second-order equations (Eq. 26) are linear equations with respect to $\eta_{2}^{ \pm}$and $\zeta_{2}^{ \pm}$. Note that equations for $\eta_{n}^{ \pm}$and $\zeta_{n}^{ \pm}$have a similar structure for each order $n$ and differ from each other only by values of their coefficients (note also that the coefficients in the equations for $\zeta_{n}^{ \pm}$have tildes). Furthermore, we omit indices " \pm " where possible, having in mind different expressions for different modes.

\section{Equations for interfaces}

In each order, we also obtain relationships between the corresponding terms in series (Eqs. 11-12). These relationships for the first-order and second-order terms are

$$
\zeta_{1}=s \eta_{1}, \quad \zeta_{2}=s \eta_{2}+s_{\text {quad }} \eta_{1}^{2}+s_{\text {disp }} \eta_{1_{\xi \xi}} x,
$$


where

$s=\left(l_{2}-2 q l_{1}\left(l_{2}-l_{1}\right)\right) / l_{1}$,

$s_{\text {quad }}=\frac{2 q\left(l_{2}-l_{1}\right)}{l_{1} H_{3}}\left(\left(\alpha^{*}+3 q\right) l_{1}-3\right)$,

$\alpha^{*}=\alpha H_{3} / c$,

$s_{\text {disp }}=\frac{\left(l_{2}-l_{1}\right) H_{3}^{2}}{6 l_{1}}\left(2 q l_{1}\left(\left(l_{2}-l_{1}\right)^{2}+12 \beta^{*}\right)-l_{2}\left(l_{1}+l_{2}\right)\right)$,

$\beta^{*}=\beta /\left(c H_{3}^{2}\right)$.

Note that $s^{ \pm}= \pm 1$ for a symmetric stratification $\left(H_{1}=\right.$ $H_{3}-H_{2}, \Delta \rho_{1}=\Delta \rho_{2}$, or $\left.r=1\right)$ analyzed in Kurkina et al. (2011b) for mode-1 waves.

Substituting $\zeta_{1}, \zeta_{2}$ into (Eq. 11), we obtain

$$
\begin{aligned}
\zeta & =\varepsilon\left(\zeta_{1}+\varepsilon \zeta_{2}\right)+O\left(\varepsilon^{3}\right) \\
& =\varepsilon s\left(\eta_{1}+\varepsilon \eta_{2}\right)+\varepsilon^{2}\left(s_{\text {quad }} \eta_{1}^{2}+s_{\text {disp }} \eta_{1_{\xi \xi}}\right)+O\left(\varepsilon^{3}\right) \\
& =s \eta+s_{\text {quad }} \eta^{2}+s_{\text {disp }} \eta_{x x}+O\left(\varepsilon^{3}\right) .
\end{aligned}
$$

Combining Eqs. (25) and (26) leads to the following generalizations of the $\mathrm{KdV}$ equation for the interfaces (presented here in the original coordinates):

$$
\begin{aligned}
& \eta_{t}+c \eta_{x}+\alpha \eta \eta_{x}+\beta \eta_{x x x}+\alpha_{1} \eta^{2} \eta_{x}+\beta_{1} \eta_{5_{x}} \\
& \quad+\gamma_{1} \eta \eta_{x x x}+\gamma_{2} \eta_{x} \eta_{x x}=0, \\
& \zeta_{t}+c \zeta_{x}+\widetilde{\alpha} \zeta \zeta_{x}+\widetilde{\beta} \zeta_{x x x}+\widetilde{\alpha}_{1} \zeta^{2} \zeta_{x}+\widetilde{\beta}_{1} \zeta_{5_{x}} \\
& \quad+\widetilde{\gamma}_{1} \zeta \zeta_{x x x}+\widetilde{\gamma}_{2} \zeta_{x} \zeta_{x x}=0 .
\end{aligned}
$$

Although Eqs. (29) and (30) can be derived separately, they are not independent. Every such derivation results in a link to the solutions of the other equation. This link is presented explicitly in Eq. (28) for the equation for the lower interface $\eta(x, t)$. It allows for a direct calculation of the disturbances for the upper interface $\zeta(x, t)$ with an accuracy of $O\left(\varepsilon^{3}\right)$. A similar link (not shown) exists for the determination of the disturbances of the lower interface using the solution for the upper interface. These couplings do not transform an equation for one interface to an equation for the other interface: e.g., substituting Eq. (28) into Eq. (30) does not yield Eq. (29), but the difference includes terms which are higher order than those retained in Eq. (29). The equation for one interface and the equation obtained by substituting the connection between the two displacements into the relationship for the other interface are only equivalent asymptotically as the small parameters go to zero. Similar interrelations become evident for a similar pair of Gardner equations below.

The coefficients of Eqs. (29) and (30) account for the main properties of the environment supporting the internal wave motion such as the location of the undisturbed interfaces, the magnitude of the density jumps between the layers and the total fluid depth. The main development here is the derivation of explicit expressions for the coefficients of these equations. The expressions for $\alpha^{ \pm}, \widetilde{\alpha}^{ \pm}, \beta^{ \pm}, \widetilde{\beta}^{ \pm}, \alpha_{1}^{ \pm}, \widetilde{\alpha}_{1}^{ \pm}$are quite complex and given in the Appendix. As the coefficients $\beta_{1}$, $\widetilde{\beta}_{1}, \gamma_{1}, \widetilde{\gamma}_{1}, \gamma_{2}, \widetilde{\gamma}_{2}$ are not analyzed in this paper, we do not provide their explicit expressions here. Note that $\beta^{ \pm} \equiv \widetilde{\beta}^{ \pm}$, $\beta_{1}^{ \pm} \equiv \widetilde{\beta}_{1}^{ \pm}$as they are just coefficients of $k^{3}$ and $k^{5}$ in the Taylor expansion at small $k$ (long-wave limit) of the dispersion relation $\omega(k)$ for linear IWs in a three-layer fluid. This dispersion relation is the same for both interfaces for a fixed mode.

The coefficients of the equation for the lower interface mode- 1 displacement $\eta^{+}$in the limits $H_{1} \rightarrow H_{2}$ or $H_{2} \rightarrow H_{3}$ are reduced into known expressions for internal waves in a two-layer fluid with density jumps $\Delta \rho_{1}+\Delta \rho_{2}$ and $\Delta \rho_{1}$, respectively. For the mode-1 displacement of the upper interface $\zeta^{+}$, the vanishing of the lowermost or middle layer (in the limiting process $H_{1} \rightarrow 0$ or $H_{1} \rightarrow H_{2}$ ) leads to the corresponding formulations for parameters of IWs in two-fluid systems with density jumps $\Delta \rho_{2}$ or $\Delta \rho_{1}+\Delta \rho_{2}$, respectively.

The similarity of the structures of the equations for disturbances of mode- 1 and mode- 2 considerably simplifies further analysis; namely, it is sufficient for solving only one of those equations (say, $\eta$ ) and then for using the abovederived relationships to obtain an approximate solution to the other equation. The relationship (Eq. 28) connecting interfacial displacements should be used when the initial conditions for Eq. (29) are being set up. A similar expression exists for $\eta(\zeta)$. This interdependence is, however, effective only during limited time intervals. The relevant nonlinear and solitarywave solutions have different wave speeds (see Eq. 24) and therefore will split over longer time intervals (considerably larger than $1 / \varepsilon$ ). This process of separation of initially basically equivalent solutions for different modes implicitly expresses the limitations of the applicability of the used asymptotic procedure.

\section{Gardner equations for interfacial displacements}

Equations (29) and (30) are integrable using the inverse scattering method only for two specific sets of nontrivial values of their coefficients (Newell, 1985; Zwillinger, 1997; Weisstein, 2002a, b). These sets, however, are not applicable to the problem of internal wave motion in stratified environments, for which this equation apparently remains nonintegrable. In this case, stationary (in a properly chosen moving coordinate system) solitary waves normally do not interact elastically. They may be generated from a suitably chosen wave system and they often radiate their energy during their motion and interact with other components of the wave field in a complicated manner (Marchant and Smyth, 1996; Osborne et al., 1998; Osborne, 2010).

It was recognized already in the first studies of internal waves using the $\mathrm{KdV}$ equation several decades ago that the coefficient at the (first order in terms of the asymptotic procedure, or quadratic in the appearance) nonlinear term in this equation may vanish or change its sign (Kakutani and Ya- 
masaki, 1978; Miles, 1979; Gear and Grimshaw, 1983). The sign of the dispersive term, however, is always the same. Importantly, the sign of the nonlinear term governs the polarity of solitary waves and soliton solutions to the relevant equation. The event of vanishing of the relevant coefficient or a change in its sign therefore leads to substantial rearrangement of the wave propagation in the vicinity of the vanishing location. A natural consequence is that an approaching soliton of, say, elevation will be transformed into a soliton of depression, and vice versa (Knickerbocker and Newell, 1980; Talipova et al., 1997; Grimshaw et al., 1999).

In order to understand how this process functions, it is necessary to build a higher-order equation that is able to describe the phenomena in the area where the coefficient at the quadratic nonlinear term vanishes. Moreover, such equations are crucial for the description of the wave motion in domains where this term is much smaller than other terms and where higher-order terms govern the wave evolution. The derivation of such equations is possible through a detailed analysis of the role of higher-order contributions (represented by coefficients $\alpha_{1}, \widetilde{\alpha}_{1}$ in Eqs. 29 and 30) of the presented asymptotic procedure into Eqs. (29) and (30) in cases when the quadratic terms vanish or are small enough to be ignored.

The relevant generalization of the $\mathrm{KdV}$ equation is called the Gardner equation. This equation contains a cubic nonlinear term, the coefficient at which may also be sign-variable, depending on the variations in the stratification (Talipova et al., 1999; Grimshaw et al., 2002, 2010; Kurkina, 2011a). If this term is negative (which is the case in a two-layer environment), the solitons' heights are limited and, in the process of reaching the limiting height, the soliton widens infinitely into a table-like disturbance. If the cubic term has a positive coefficient, the Gardner equation possesses several types of solitons with different polarities.

If the quadratic and cubic terms of Eqs. (29) and (30) have the same order of magnitude, the above-described asymptotic procedure is not applicable. A possibility is to make explicit use of the smallness of the coefficient at the quadratic nonlinear term and to assume that $\alpha \sim \delta$, where $\delta \ll 1$. A suitable procedure may be constructed using asymptotic expansions similar to Eqs. (10) and (11) but built for two independent small parameters $\mu$ and $\varepsilon$ (see, e.g., Lamb and Yan, 1996). It is now necessary to account for second-order terms with respect to both parameters. This can be done by re-expanding the above series in terms of $\alpha \sim \delta$. A meaningful evolution equation can then be obtained using the condition that three terms (quadratic and cubic nonlinearity, and the leading term responsible for linear dispersion) are of the same order of magnitude. This is possible if $\delta \sim \varepsilon$ and $\mu \sim \varepsilon^{2}$.

The procedure itself is similar to the above-described technique, and sorting out the terms with similar powers of $\varepsilon$ leads to the desired evolution equations. These equations, at the lowest order, for the disturbances at the interfaces of the three-layer environment are equivalent to a pair of interre-

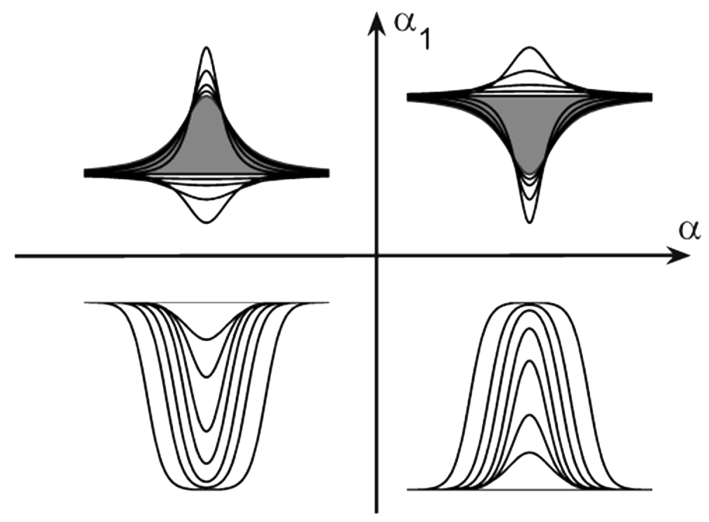

Figure 2. Shapes of soliton solutions to the Gardner equation for different combinations of the signs of coefficients at its nonlinear terms (idea of representation by R. Grimshaw, E. Pelinovsky and T. Talipova).

lated Gardner equations:

$\eta_{t}+c \eta_{x}+\alpha \eta \eta_{x}+\alpha_{1} \eta^{2} \eta_{x}+\beta \eta_{x x x}=0$,

$\zeta_{t}+c \zeta_{x}+\widetilde{\alpha} \zeta \zeta_{x}+\widetilde{\alpha}_{1} \zeta^{2} \zeta_{x}+\beta \zeta_{x x x}=0$

Formally, they only differ from Eqs. (29) and (30) by the absence of terms $\beta_{1} \eta_{5 x}, \gamma_{1} \eta \eta_{x x x}$ and $\gamma_{2} \eta_{x} \eta_{x x}$. The actual difference is, however, much deeper, as Eq. (31) expresses the dynamics on another scale, governed by two independent small parameters. This difference becomes to some extent evident via another relationship between the soliton amplitude and its width. These equations govern the interfacial motion of weakly nonlinear finite-amplitude unidirectional waves neglecting mode interactions. Their coefficients are called environmental parameters, because they account for the background conditions (configuration of the medium). These coefficients are functions of the layers' positions and density jumps between the layers (or their ratio $r$ ).

Similarly to Eqs. (29) and (30), Eq. (31) is interrelated within the accuracy of $O\left(\varepsilon^{3}\right)$ :

$\zeta=s \eta+s_{\text {quad }} \eta^{2}$.

An inverse of Eq. (32), $\eta=\eta(\zeta)$, can be derived similarly in a straightforward manner.

The analytical one-soliton solution of the Gardner equation is well known (Helfrich and Melville, 2006; Pelinovsky et al., 2007):

$\eta(x, t)=\frac{A}{1+B \cosh (\gamma(x-V t))}$.

The soliton velocity $V=c+\beta \gamma^{2}$ is expressed through the inverse width of the soliton $\gamma$. The parameters $A$ and $B$ depend on the coefficients of Eq. (31) and determine the soliton 
amplitude $a$ as the extreme value of the function $\eta(x, t)$ in Eq. (33):

$a=\frac{A}{1+B}, \quad A=\frac{6 \beta \gamma^{2}}{\alpha}, \quad B^{2}=1+\frac{6 \alpha_{1} \beta \gamma^{2}}{\alpha^{2}}$.

The parameters of the family of solutions can also be expressed through its amplitude $a$ :

$\gamma^{2}=a \frac{2 \alpha+a \alpha_{1}}{6 \beta}, \quad A=a\left(2+a \frac{\alpha_{1}}{\alpha}\right), \quad B=1+a \frac{\alpha_{1}}{\alpha}$.

There are different branches of the soliton solutions depending on the signs of coefficients at the nonlinear terms; see Fig. 2. The asymptotics of the solution (Eq. 33) is described for instance in Pelinovsky et al. (2007).

\section{Environmental parameters of IWs}

Whilst the mathematical properties of the Gardner equation and its solutions are quite well known, these properties may vary radically along the propagation of realistic IWs in natural environments (Nakoulima et al., 2004). Therefore, it is important to analyze the behavior of the environmental parameters for possible ranges of parameters of the medium, which govern the kinematic and nonlinear characteristics of the IW field. The above-described explicit expressions for the coefficients of Eqs. (29) and (30) (see Appendix) remain valid also for Eq. (31) and allow the production of the estimates of nonlinear IW shapes, limiting amplitudes and projections of possible transformations of solitary IWs in an inhomogeneous medium (Kurkina et al., 2011a).

To describe the behavior of the environmental parameters, we use their graphs on the "phase" plane of scaled undisturbed interface positions $\left(l_{1}=H_{1} / H_{3}, l_{2}=H_{2} / H_{3}\right)$ for the fixed value of the ratio $r$ of density jumps. Along the diagonal $l_{1}=l_{2}$, the two interfaces coincide, and this line represents a two-layer fluid. The other diagonal $l_{1}=1-l_{2}$ corresponds to the symmetric geometric configuration of layers that has been addressed in detail in Kurkina et al. (2011b). As we always require $l_{2} \geq l_{1}$, the triangle to the left of the diagonal $l_{2}=l_{1}$ represents all possible ranges of $l_{1}$ and $l_{2}$. Therefore, we use this triangle to analyze the environmental parameters for the upper interface (coefficients in the equations for $\left.\zeta^{ \pm}\right)$. For a Boussinesq fluid, there is symmetry: switching the depths of the upper and lower layers and interchanging the density jumps will result in exactly the same behavior with the waves flipped over. The coefficients for the equation for $\eta$ given in terms of $l_{1}$ and $l_{2}$ should be the same as the equations for $\zeta$ given in terms of $1-l_{2}$ and $1-l_{1}$ and replacing $r$ with $1 / r$. When the Boussinesq approximation is involved, no preferred vertical direction exists; therefore, if the solution of a fixed polarity exists in this framework, its mirror disturbance is also a solution.
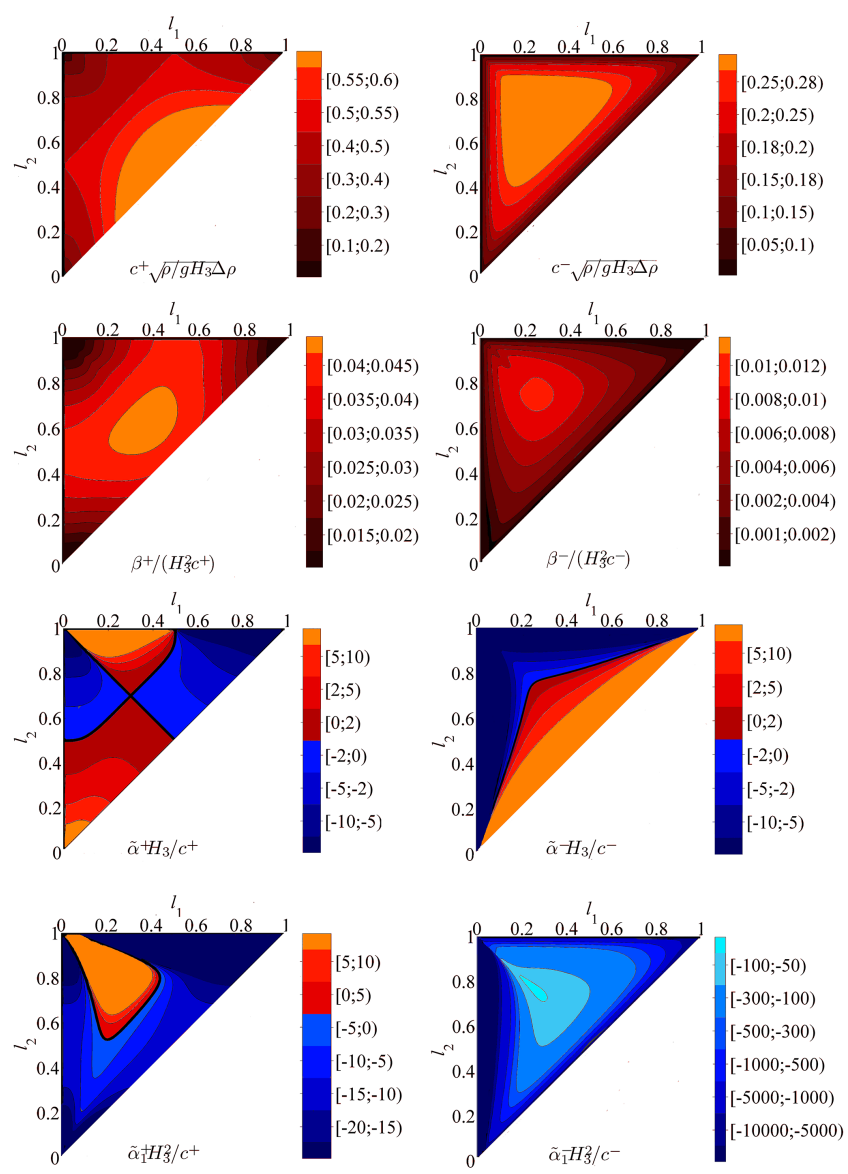

Figure 3. Variation in environmental parameters for IWs of fast (left column) and slow (right column) modes in the particular case $r=1$ $\left(\Delta \rho_{1}=\Delta \rho_{2}\right)$. Zero contours are given by bold black lines.

Figure 3 shows the maps of values of parameters $c^{ \pm}, \beta^{ \pm}$, $\widetilde{\alpha}^{ \pm}, \widetilde{\alpha}_{1}^{ \pm}$as functions of $l_{1}$ and $l_{2}$ when $r=\Delta \rho_{2} / \Delta \rho_{1}=1$. The parameters $c^{ \pm}$and $\beta^{ \pm}$are always positive for the threelayer fluid. They tend to zero only when one of the layers vanishes for mode- 2 waves $\left(c^{-}, \beta^{-}\right)$or when the thicknesses of two out of the three layers simultaneously tend to zero for mode- 1 waves $\left(c^{+}, \beta^{+}\right)$. The parameters $c^{-}$and $\beta^{-}$both have one maximum for the symmetric configuration $l_{2}=1-l_{1}=3 / 4$. Their counterparts $c^{+}$and $\beta^{+}$have the maxima at the point $l_{1}=l_{2}=1 / 2$, i.e., when the three-layer fluid degenerates into a two-layer fluid with equal layers and with a density jump of $2 \Delta \rho$.

A specific feature of the coefficients at the quadratic nonlinear term $\alpha^{ \pm}, \widetilde{\alpha}^{ \pm}$is that they may vanish for some nondegenerate three-layer configurations. These parameters for mode- 1 IWs vanish along the diagonal $l_{2}=1-l_{1}$ due to the symmetry of the wave motion. They also have other coinciding zero contours passing through the points $(0 ; 0.5)$, $(0.3 ; 0.7),(0.5 ; 1)$ on the plane $\left(l_{1}, l_{2}\right)$. The signs of $\alpha^{+}$and $\widetilde{\alpha}^{+}$always coincide. Each of the quadratic nonlinear coefficients $\alpha^{-}$and $\widetilde{\alpha}^{-}$for mode-2 IWs has a smooth zero contour 


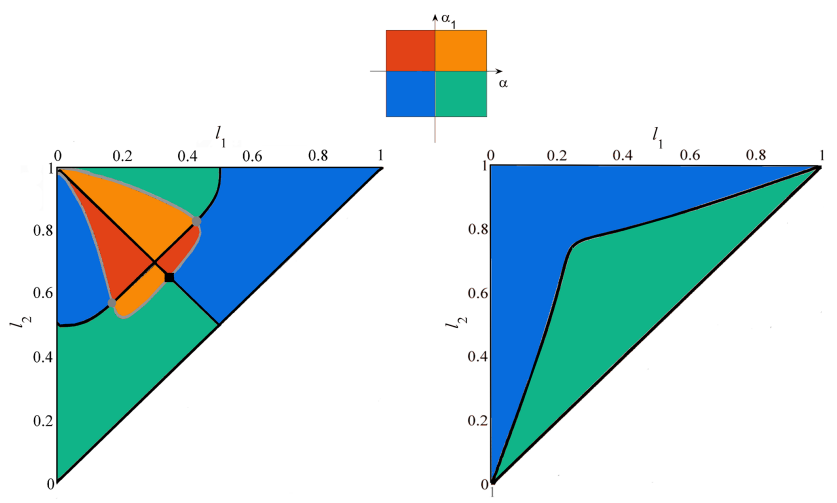

Figure 4. Map of the appearance of possible soliton solutions to the Gardner equation for a three-layer fluid for fast (left panel) and slow (right panel) internal wave modes in the particular case $r=1\left(\Delta \rho_{1}=\Delta \rho_{2}\right)$. The legend of patterns is given at the top. Bold black curves correspond to $\widetilde{\alpha}=0$. Bold grey curves correspond to $\widetilde{\alpha}_{1}=0$. The markers show points at which $\widetilde{\alpha}=\widetilde{\alpha}_{1}=0$. Black squares indicate the combinations $\left(l_{1}, l_{2}\right)=(9 / 26 ; 17 / 26)$ $\approx(0.3462 ; 0.6538)$, and grey circles the combinations $\left(l_{1}, l_{2}\right) \approx$ $(0.1703 ; 0.5717)$ and $\left(l_{1}, l_{2}\right) \approx(0.4283 ; 0.8297)$.

passing through the points $(0 ; 0),(0.25 ; 0.75),(1 ; 1)$ on the plane $\left(l_{1}, l_{2}\right)$. The signs of these parameters, determining the polarity of solitary IWs in the KdV approximation, are always opposite, reflecting the structure of mode- 2 waves that always create oppositely directed interface displacements.

The coefficients at the cubic nonlinear terms $\alpha_{1}^{ \pm}, \widetilde{\alpha}_{1}^{ \pm}$behave differently for IWs of different modes (Fig. 4). The coefficients $\alpha_{1}^{+}$and $\widetilde{\alpha}_{1}^{+}$in the Gardner equation for mode-1 IWs have two distinct closed zero contours passing through the points $(0 ; 1)$ and $(9 / 26 ; 17 / 26)$ on the plane $\left(l_{1}, l_{2}\right)$. The latter point corresponds to the symmetric stratification. The possibility of the change in the sign of the cubic nonlinear parameter at this point for IWs in a three-layer fluid was first mentioned in Grimshaw et al. (1997). For such a stratification, both coefficients at the leading nonlinear terms in the weakly nonlinear theory for mode-1 IWs vanish simultaneously. The wave dynamics near such a regime is described by an extended $(2+4) \mathrm{KdV}$ equation (Kurkina et al., 2011b). There exist two other such points (Fig. 4) in each half-plane. The parameters $\alpha_{1}^{-}$and $\widetilde{\alpha}_{1}^{-}$of mode- 2 waves are always negative. Their minimal absolute values occur for a symmetric stratification, when all layers are relatively thick. When one of the layers vanishes, $\alpha_{1}^{-}$and $\widetilde{\alpha}_{1}^{-}$tend to negative infinity, reflecting the fact that the three-layer mode- 2 wave regime does not have a valid asymptotic process towards a two-layer dynamics.

For both modes, the absolute values of the coefficients at the quadratic and cubic terms of the equations for the upper and lower interfaces are not equal except if they are zero or if the stratification is symmetric. This feature exemplifies the asymmetry of the interfacial displacements for asymmetric three-layer stratifications.
Figure 4 illustrates possible soliton branches of IWs of both modes for $r=\Delta \rho_{2} / \Delta \rho_{1}=1$. It is clear that all possible regimes shown in Fig. 2 can be implemented for mode-1 waves. Waves of both polarities can exist (waves of elevation on both interfaces or waves of depression on both interfaces), but properties of such waves can be different for different combinations of the parameters of the medium. The left panel of Fig. 4 also presents information about all points at which both the coefficients at the cubic and quadratic terms are zero. Higher-order extensions of the weakly nonlinear models have to be produced to provide a balance between the nonlinear and dispersive terms and to describe IWs in the vicinity of such points properly. These models for the points corresponding to asymmetric stratifications (and not located at the diagonal of the $\left(l_{1}, l_{2}\right)$ plane) are supposed to be similar to those described in Kurkina et al. (2011b) for a symmetric situation.

The pool of solitonic mode-2 waves (Fig. 4, left panel) consists of solitons with a limited amplitude. As described above, these solitons broaden while approaching this limit and form table-like structures. Their polarity on each interface is determined by the sign of the coefficient at quadratic nonlinear terms $\alpha_{1}^{-}$and $\widetilde{\alpha}_{1}^{-}$. Thus, two types of mode- 2 waves can exist in the three-layer fluid: convex waves displacing the upper interface upward and the lower interface downward, and concave waves doing the opposite. Both types of mode-2 IWs were observed in the South China Sea (Yang et al., 2010), but concave waves occur fairly rarely. Convex waves were found much more often. They were experimentally observed in Mehta et al. (2002) and their nature can be explained as an intrusion of water into the middle layer.

The fluid at the mid-depth of a symmetric three-layer fluid (with equal density jumps) is not displaced in the case of mode-2 waves (Lamb, 2006), so a rigid boundary placed at the mid-depth will not be affected by the flow. The solution in the upper or lower half of the fluid is simply the solution for a two-layer fluid involving half of the middle layer; these "partial" solutions of course only exist together, and must be synchronized. The middle layer plays the role of a fluid that both underlies the upper layer and overlies the lower layer. If half of the middle layer is shallower than the lower and upper layers, a hump of elevation may exist at the upper interface, and a hump of depression at the lower one. This feature was used to interpret mode- 2 solitary IWs experimentally observed by Mehta et al. (2002) with the help of the Gardner model for a two-layer fluid.

A summary of the results of some calculations of fully nonlinear and higher-order weakly nonlinear solitary IWs is presented in Fig. 5. These data are in qualitative agreement with the predictions of the model based on the Gardner equation. Some differences can be explained by the use of smoothed three-layer stratifications in numerical calculations in Lamb (2006). 


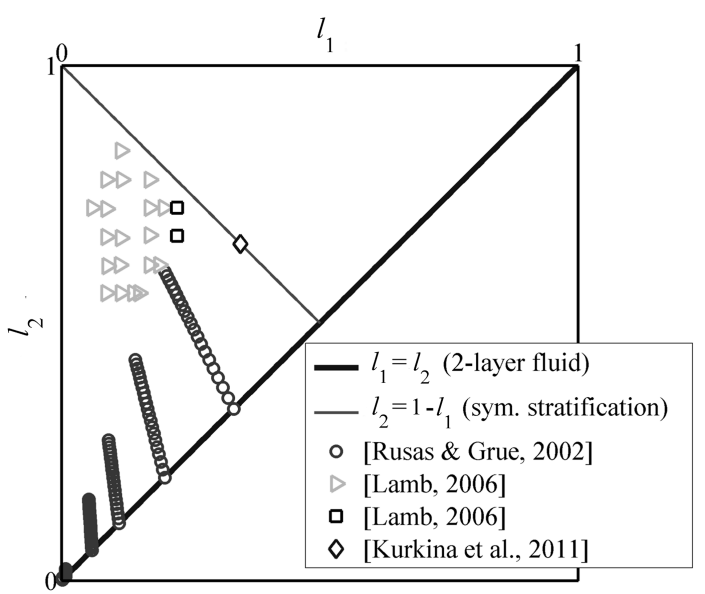

Figure 5. Points corresponding to stratifications for which mode1 ISWs were considered in Boussinesq approximation with equal density jumps in different approaches. Circles: positive polarity waves (fully nonlinear numerical method for ISWs in a three-layer fluid) (Rusås and Grue, 2002). Triangles: waves of elevation with a minimum amplitude greater than zero; squares: waves of elevation with no minimum amplitude (fully nonlinear numerical model, continuous almost three-layerstratification) (Lamb, 2006). Left vicinity of the diamond on the line $l_{2}=1-l_{1}$ : waves of both polarities broaden and flatten while approaching an amplitude limit, $(2+4)$ $\mathrm{KdV}$ model for IWs in a symmetric three-layer fluid (Kurkina et al., 2011a, 2012).

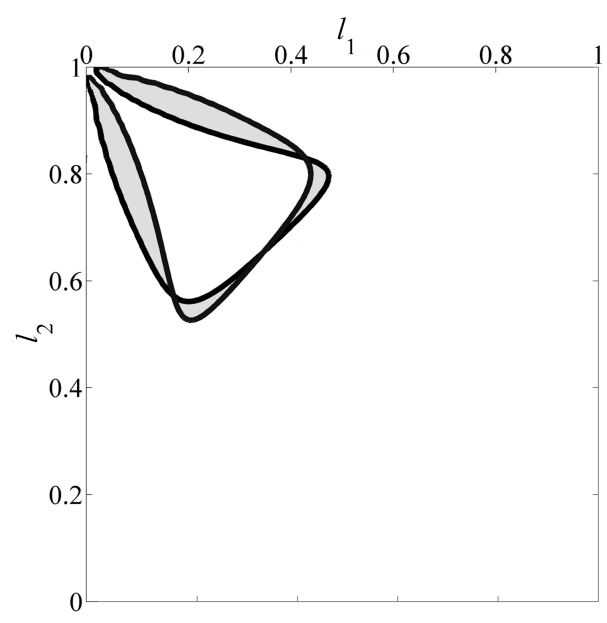

Figure 6. Comparison of curves $\alpha_{1}^{+}=0$ and $\widetilde{\alpha}_{1}^{+}=0$ when $\Delta \rho_{1}=$ $\Delta \rho_{2}$. The intersection points are the same as marked points in Fig. 5, left panel, where $\alpha^{+}=0$ and $\widetilde{\alpha}^{+}=0$. The shaded regions represent the configurations where the displacements of the interfaces belong to different branches of the solution of Eq. (33) shown in Fig. 2.

The contours of $\alpha_{1}^{+}=0$ and $\widetilde{\alpha}_{1}^{+}=0$ in Gardner equations for the lower and the upper interface, respectively, are shown in Fig. 6 for the case $\Delta \rho_{1}=\Delta \rho_{2}$. The difference between zero contours as shown in Fig. 6 is related to the fact that the second-order coefficients are not uniquely determined until some choice is made as to what the dependent variable in the weakly nonlinear equation represents: it could be the displacement of the lower interface, the upper interface, the average of the two, the velocity at some level between the two interfaces, etc. This was discussed, for example, in Lamb and Yan (1996). While the first-order coefficients are not affected by this choice, the second-order coefficients are. As the signs of $\alpha^{+}$and $\widetilde{\alpha}^{+}$are always the same for any fixed point of the plane $\left(l_{1}, l_{2}\right)$, both of these solutions are taken from the left half-plane $\left(\alpha, \alpha_{1}\right)$ or both from the right half-plane. So, different choices give different predictions when we are close to critical values where the cubic nonlinear coefficient is zero. For the fully nonlinear dispersive equations, we will only be in one regime.

The waves from mode-1 produce disturbances of the interfaces of the same polarity. Therefore, if $\alpha_{1}>0$, the polarity of the solution matches the sign of $\alpha$. The shaded areas in Fig. 6 (the configurations where the displacements of the interfaces belong to different branches of the solution; Eq. 33) are located fairly close to the contours $\alpha_{1}^{+}=0, \widetilde{\alpha}_{1}^{+}=0$. Consequently, the absolute values of the coefficients $\alpha_{1}^{+}$and $\widetilde{\alpha}_{1}^{+}$ are small in these areas, and the corresponding solution apparently resembles the KdV soliton. This regime is the limiting one for the solutions corresponding to both "upper" and "lower" families of small-amplitude solitons depicted in Fig. 2. This feature confirms the correctness of the presented constructions and the applicability of the solutions $\left(\eta^{+}, \zeta^{+}\right)$ to internal waves of mode-1, even in these areas.

In this case, it should be decided to derive a Gardner equation for the lower interface or for the upper interface. The fact that the relationship (Eq. 32) does not convert the second of Eq. (31) to the first of Eq. (31) is connected to the fact that deriving the Gardner equation for the upper interface amounts to a different choice and hence results in different coefficients. As for stratifications with the same density jump across each interface, the interface furthest to the middepth is, probably, the best choice for the dependent variable in the Gardner equation. In this case, one would use the equation for $\zeta$ and the relationship connecting interfacial displacements if $l_{1}<1-l_{2}$ and the equation for $\eta$ with connection Eq. (32) otherwise. For different density jumps, the interface with the largest density jump should be used.

In the seas and oceans, the assertion $r=\Delta \rho_{2} / \Delta \rho_{1}=1$ is normally not valid, and the magnitudes of density jumps at different interfaces can be considerably different. To depict the changes associated with the above "phase diagrams" of various solutions, we chose the values $r=2$ and $r=1 / 2$ to characterize the variations in the coefficients in question and the wave propagation regimes. Different values of $r$ significantly influence the patterns for mode- 1 environmental parameters. The modifications are the largest for the values of the coefficients at the nonlinear terms. These changes obviously affect the possible regimes for soliton appearance and propagation (Figs. 7 and 8). Interestingly, the parameters 

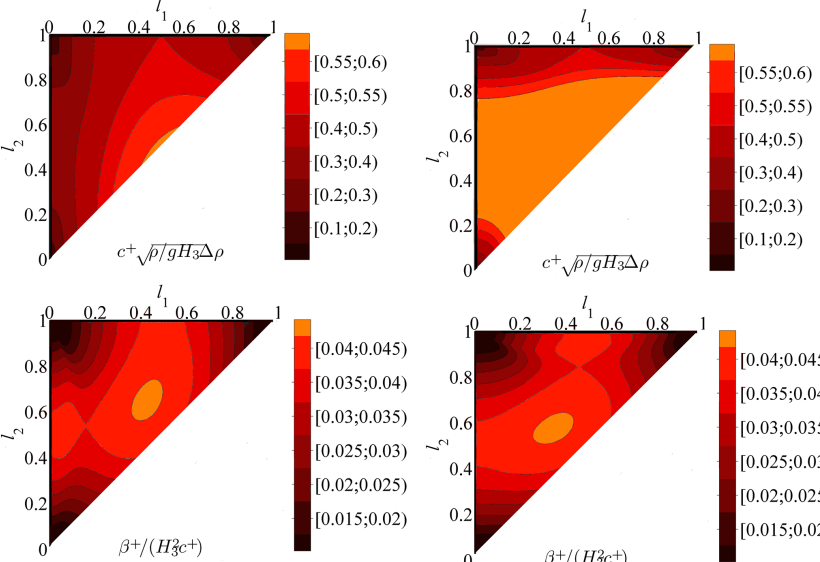

$0.4^{l_{1}} 0.6 \quad 0.8$
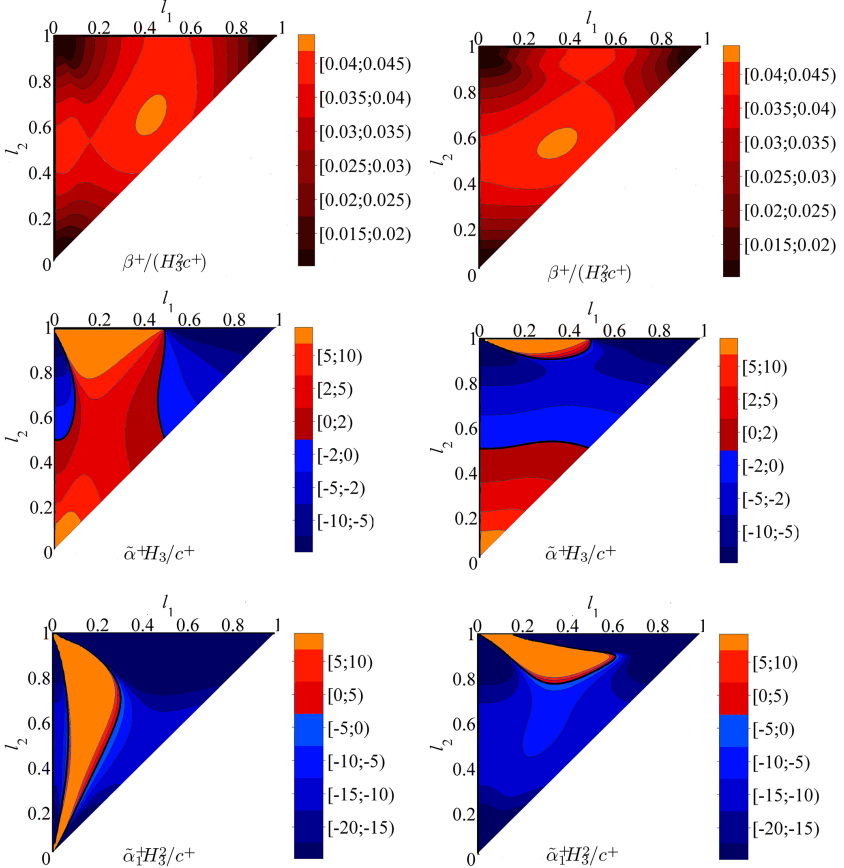
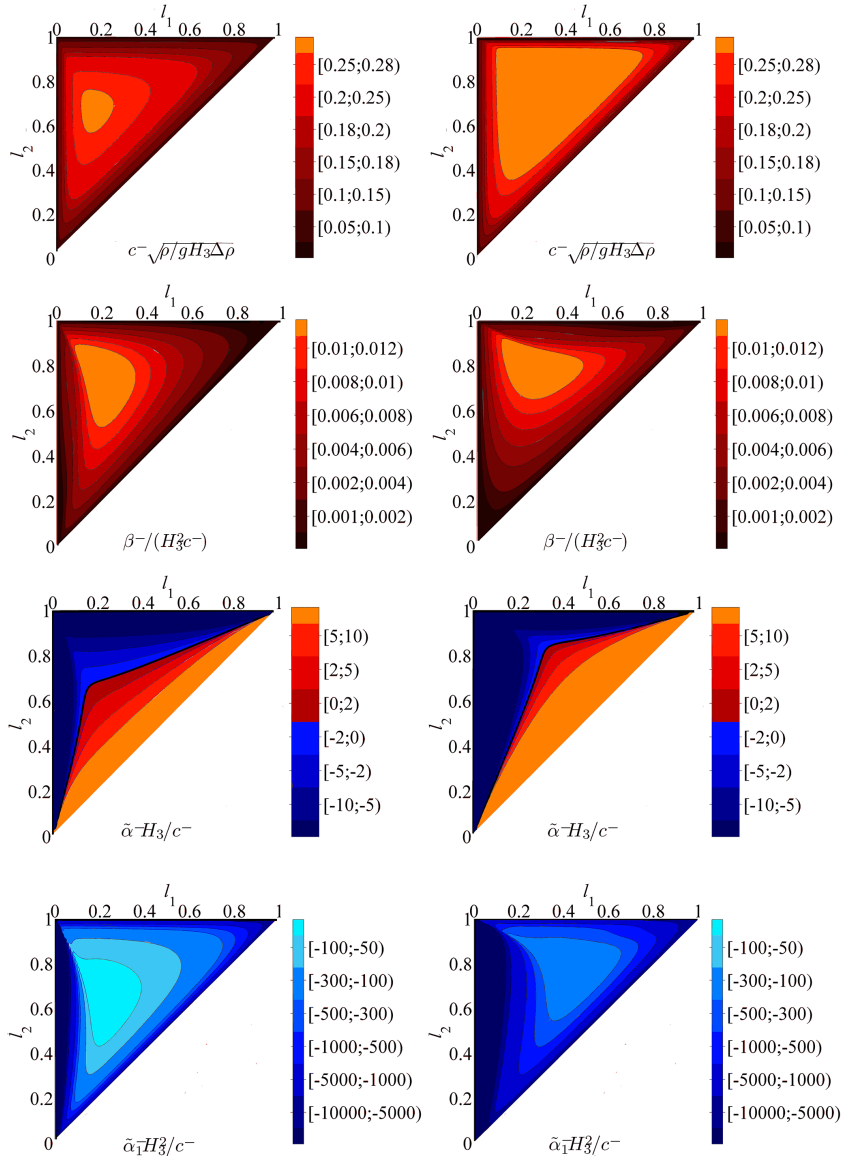

Figure 9. Variation in the environmental parameters for mode-2 IWs in a three-layer fluid with unequal density jumps at the interfaces. Left column: $\Delta \rho_{1}=2 \Delta \rho_{2}$. Right column: $\Delta \rho_{1}=\Delta \rho_{2} / 2$.
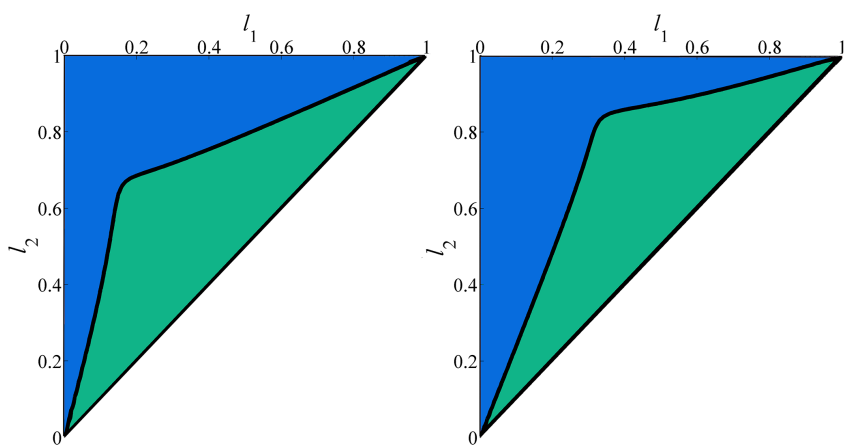

Figure 10. Map of the appearance of possible soliton solutions for mode-2 IWs in a three-layer fluid with unequal density jumps at the interfaces. Left column: $\Delta \rho_{1}=2 \Delta \rho_{2}$. Right column: $\Delta \rho_{1}=$ $\Delta \rho_{2} / 2$.

$\frac{1}{2} \Delta \rho_{2} \tanh \left(\left(z-H_{2}\right) / d_{2}\right)$ for small $d_{1}, d_{2}$ could be modeled numerically with a smoothed three-layer model, the considered three-layer model completely misses this. 


\section{Conclusions}

The performed analytical investigation of equations governing the propagation of weakly nonlinear internal waves in a relatively simple but frequently occurring in nature and rich in content three-layer environment has highlighted several interesting features that distinguish this environment from the similar but symmetric case (Kurkina et al., 2011b). We have derived a system of nonlinear evolution equations for long, finite-amplitude internal waves, valid for both possible modes of wave motion at the interfaces of a three-layer fluid of arbitrary ratios of the depths between the layers and arbitrary (but small) values of the density jumps at the interfaces in the commonly used framework of Boussinesq approximation. Although each of the equations of this system is known, the analysis of interrelations of the equations for disturbances at different interfaces and the description of the possible wave propagation regimes provides new insight into the dynamics of internal waves in natural environments.

As expected, the classical Korteweg-de Vries equation is only conditionally a proper tool for the description of the internal wave motion, because the coefficient at its nonlinear (quadratic) term may vanish for certain combinations of the environmental parameters (layer depths and densities) of the three-layer fluid. This shortage affects the description of both mode- 1 and mode- 2 waves (with the same and opposite polarities of the disturbances to the interfaces, respectively). To resolve such situations, the derivation of the evolution equations is extended by systematically accounting for the next (second) order in magnitude terms in the relevant asymptotic procedure. This procedure leads to two implicitly interrelated Gardner equations (one for each interface) with possibly spatially varying coefficients.
The derived explicit analytical (algebraic) expressions for their coefficients as functions of the properties of the fluid made it possible to perform full analysis of the possible appearances, propagation regimes and transformation domains of solitary solutions of both modes to these equations in horizontally inhomogeneous environments. In particular, we perform a comprehensive analysis of the situations in which the coefficients of the quadratic and cubic nonlinearity simultaneously vanish. This may happen for three different combinations of the properties of the fluid. On such occasions, Gardner equations fail to describe the internal wave dynamics, and it is necessary to extend the derivation of the proper evolution equation to even higher-order terms. While the relevant analysis has recently been performed for the special case of a symmetric three-layer fluid (Kurkina et al., 2011b), the multitude of options for the failure of the Gardner equation to describe the dynamics of three-layer flows calls for further in-depth analysis of this problem. More generally, the situations corresponding to any line representing vanishing coefficients in Fig. 5 require deeper analysis of the associated dynamics. 


\section{Appendix A}

Coefficients of Eqs. (29), (30) as well as the Gardner equations, namely, Eq. (31) for interfacial waves of both modes propagating on both interfaces.

$$
\begin{aligned}
& \frac{\beta^{ \pm}}{c^{ \pm} H_{3}^{2}}=\frac{\widetilde{\beta}^{ \pm}}{c^{ \pm} H_{3}^{2}}= \\
& 12 \frac{2 r q^{ \pm}\left(1-l_{2}\right)\left(l_{2}-l_{1}\right)\left(2 l_{1}\left(l_{2}-l_{1}\right)^{2} q^{ \pm}-l_{2}\left(l_{2}+l_{1}\right)\right)+2 q^{ \pm} l_{1}\left(l_{1}^{3}-3 l_{1}^{2}+\left(3-\left(1-l_{2}\right)^{2}\right) l_{1}-2 l_{2}\left(2-l_{2}\right)\right)-l_{1}^{2}+l_{2}\left(2-l_{2}\right)}{1-4 r q^{ \pm 2} l_{1}\left(1-l_{2}\right)\left(l_{2}-l_{1}\right)} \\
& \frac{\alpha^{ \pm} H_{3}}{c^{ \pm}}=\frac{3}{2\left(1-l_{2}\right) l_{1}} \frac{8 r q^{ \pm 2} l_{1}\left(1-l_{2}\right)^{2}\left(l_{2}-l_{1}\right)\left(l_{1} q^{ \pm}-1\right)-4 q^{ \pm 2} l_{1}^{2}\left(l_{1}^{2}-\left(1+l_{2}\right) l_{1}+l_{2}\right)+4 q^{ \pm} l_{1}\left(l_{2}-l_{1}\right)-2 l_{2}+1}{1-4 r q^{ \pm 2} l_{1}\left(1-l_{2}\right)\left(l_{2}-l_{1}\right)} \\
& \alpha_{1}^{ \pm}=\frac{1}{2\left(1-l_{2}\right)^{2} H_{3}^{2} c^{ \pm} l_{1}^{2}\left(1-4 r q^{ \pm 2}\left(1-l_{2}\right) l_{1}\left(l_{2}-l_{1}\right)\right)} \times\left(\alpha^{ \pm 2} H_{3}^{2} l_{1}^{2}\left(1-l_{2}\right)^{2}\left(-12 r q^{ \pm 2} l_{1}\left(1-l_{2}\right)\left(l_{2}-l_{1}\right)-1\right)\right) \\
& +\alpha^{ \pm} H_{3} c^{ \pm}\left(1-l_{2}\right) l_{1}\left(4 r q^{ \pm 2}\left(1-l_{2}\right)^{2}\left(l_{2}-l_{1}\right) l_{1}\left(-20 l_{1} q^{ \pm}+11\right)+16 q^{ \pm 2} l_{1}^{2}\left(1-l_{1}\right)\left(l_{2}-l_{1}\right)+2 q^{ \pm} l_{1}\left(l_{2}-l_{1}\right)+5\left(1-2 l_{2}\right)\right) \\
& +12 r q^{ \pm 2} c^{ \pm 2}\left(1-l_{2}\right)^{3}\left(l_{2}-l_{1}\right) l_{1}\left(-10 q^{ \pm 2} l_{1}^{2}+15 q^{ \pm} l_{1}-3\right) \\
& +12 q^{ \pm 3} c^{ \pm 2} l_{1}^{3}\left(-4 l_{1}^{3}+3 l_{1}^{2}\left(3+l_{2}\right)+l_{1}\left(\left(1-l_{2}\right)^{2}+6\left(1-l_{2}\right)-12\right)+\left(5-l_{2}\right) l_{2}\right) \\
& -18 q^{ \pm 2} c^{ \pm 2} l_{1}^{2}\left(2 l_{1}^{2}\left(3-l_{2}\right)+l_{1}\left(2\left(1-l_{2}\right)^{2}+\left(1-l_{2}\right)-8\right)+l_{2}\left(5-l_{2}\right)\right)+18 q^{ \pm} c^{ \pm 2} l_{1}\left(1+l_{2}\right)\left(l_{2}-l_{1}\right) \\
& \left.-6 c^{ \pm 2}\left(3\left(1-l_{2}\right)^{2}-3\left(1-l_{2}\right)+1\right)\right)
\end{aligned}
$$

$\gamma_{1}^{ \pm}=\frac{1}{1} 2 c^{ \pm} H_{3} l_{1}\left(1-l_{2}\right)\left(1-4 r q^{ \pm 2} l_{1}\left(1-l_{2}\right)\left(l_{2}-l_{1}\right)\right) \times\left(\left(144 r q^{ \pm} H_{3} l_{1}^{2}\left(1-l_{2}\right)^{2}\left(l_{1}-l_{2}\right)-12 H_{3} l_{1}\left(1-l_{2}\right)\right) \beta^{ \pm}\right.$

$\left.+\left(\left(8 H_{3}^{3} l_{1}^{2} c^{ \pm}\left(1-l_{2}\right)^{2}\left(l_{1}^{3}-l_{2}^{3}\right)+24 H_{3}^{3} l_{1}^{3} c^{ \pm}\left(1-l_{2}\right)^{2}\left(l_{1}-l_{2}\right)\right)\right) r q^{ \pm}-2 H_{3}^{3} l_{1} c^{ \pm}\left(1-l_{2}\right)\left(l_{1}^{2}-l_{2}^{2}\right)\right) \alpha^{ \pm}$

$+\left(384 l_{1}^{2} c^{ \pm} q^{ \pm 3} r\left(1-l_{2}\right)^{2}\left(l_{1}-l_{2}\right)-240 l_{1} c^{ \pm} q^{ \pm 2} r\left(1-l_{2}\right)^{2}\left(l_{1}-l_{2}\right)+48 l_{1}^{2} c^{ \pm} q^{ \pm 2}\left(l_{1}^{2}-l_{1}\left(1+l_{2}\right)+l_{2}\right)\right.$

$\left.24 c^{ \pm}\left(q^{ \pm} l_{1}\left(l_{2}-l_{1}\right)+1+2 l_{2}\right)\right) \beta^{ \pm}+\left(40 H_{3}^{2} c^{ \pm 2}\left(1-l_{2}\right)^{2}\left(q^{ \pm 3} l_{1}^{2}\left(l_{1}^{3}-l_{2}^{3}\right)-q^{ \pm 2} l_{1}\left(l_{1}^{3}-l_{2}^{3}\right)\right)+120 H_{3}^{2} c^{ \pm 2}\left(1-l_{2}\right)^{2}\right.$

$\left.\times q^{ \pm 3} l_{1}^{3}\left(l_{2}^{2}-l_{2} l_{1}\right)-96 H_{3}^{2} c^{ \pm 2} q^{ \pm 2}\left(1-l_{2}\right)^{2} l_{1}^{2}\left(l_{2}^{2}-l_{2} l_{1}\right)+4 H_{3}^{2} c^{ \pm 2} q^{ \pm}\left(1-l_{2}\right)^{2}\left(l_{2} l_{1}^{2}-l_{1}^{3}\right)\right) r+\left(12 l_{1}^{4}\right.$

$-4 l_{1}^{3}\left(5+7 l_{2}\right)+4 l_{1}^{2}\left(11\left(1-l_{2}\right)^{2}-3-21 l_{2}\right)+4 l_{1}\left(7\left(1-l_{2}\right)^{3}-22\left(1-l_{2}\right)^{2}+21\left(1-l_{2}\right)-12\right)$

$\left.-4 l_{2}\left(7\left(1-l_{2}\right)^{2}-4\left(1-l_{2}\right)+3\right)\right) H_{3}^{2} c^{ \pm 2} q^{ \pm 2} l_{1}^{2}+\left(20 l_{1}^{3}\left(1-l_{2}\right)-12 l_{1}^{2}\left(3-2 l_{2}\right)-4 l_{1}\left(5\left(1-l_{2}\right)^{3}\right.\right.$

$\left.\left.-11\left(1-l_{2}\right)^{2}+6\left(1-l_{2}\right)-6\right) H_{3}^{2} c^{ \pm 2} q^{ \pm} l_{1}+\left(l_{1}^{2}\left(1-4 l_{2}\right)-l_{2}^{2}\left(1-4 l_{2}\right)\right) H_{3}^{2} c^{ \pm 2}\right)$ 


$$
\begin{aligned}
& \gamma_{2}^{ \pm}=\frac{1}{12 c^{ \pm} H_{3} l_{1}\left(1-l_{2}\right)\left(1-4 r q^{ \pm 2} l_{1}\left(1-l_{2}\right)\left(l_{2}-l_{1}\right)\right)} \times\left(\left(216 r q^{ \pm} H_{3} l_{1}^{2}\left(1-l_{2}\right)^{2}\left(l_{1}-l_{2}\right)-18 H_{3} l_{1}\left(1-l_{2}\right)\right) \beta^{ \pm}\right. \\
& +\left(\left(24 H_{3}^{3} l_{1}^{2} c^{ \pm}\left(1-l_{2}\right)^{2}\left(l_{1}^{3}-l_{2}^{3}\right)+72 H_{3}^{3} l_{1}^{3} c^{ \pm}\left(1-l_{2}\right)^{2}\left(l_{1}-l_{2}\right)\right) r q^{ \pm}-6 H_{3}^{3} l_{1} c^{ \pm}\left(1-l_{2}\right)\left(l_{1}^{2}-l_{2}^{2}\right)\right) \alpha^{ \pm} \\
& +\left(192 l_{1}^{2} c^{ \pm} q^{ \pm 3} r\left(1-l_{2}\right)^{2}\left(l_{1}-l_{2}\right)-48 l_{1} c^{ \pm} q^{ \pm 2} r\left(1-l_{2}\right)^{2}\left(l_{1}-l_{2}\right)+96 l_{1}^{2} c^{ \pm} q^{ \pm 2}\left(l_{1}^{2}-l_{1}\left(1+l_{2}\right)+l_{2}\right)\right. \\
& \left.+12 c^{ \pm}\left(2 q^{ \pm} l_{1}\left(l_{2}-l_{1}\right)+1+2 l_{2}\right)\right) \beta^{ \pm}+\left(56 H_{3}^{2} c^{ \pm 2}\left(1-l_{2}\right)^{2}\left(q^{ \pm 3} l_{1}^{2}\left(l_{1}^{3}-l_{2}^{3}\right)-q^{ \pm 2} l_{1}\left(\frac{13}{7} l_{1}^{3}-l_{2}^{3}\right)\right)+168 H_{3}^{2} c^{ \pm 2}\left(1-l_{2}\right)^{2}\right. \\
& \left.\times q^{ \pm 3} l_{1}^{3}\left(l_{2}^{2}-l_{2} l_{1}\right)-96 H_{3}^{2} c^{ \pm 2} q^{ \pm 2}\left(1-l_{2}\right)^{2} l_{1}^{2}\left(\frac{5}{2} l_{2}^{2}-l_{2} l_{1}\right)-4 H_{3}^{2} c^{ \pm 2} q^{ \pm}\left(1-l_{2}\right)^{2}\left(l_{2} l_{1}^{2}-l_{1}^{3}\right)\right) r+\left(12 l_{1}^{4}\right. \\
& -4 l_{1}^{3}\left(7+5 l_{2}\right)+4 l_{1}^{2}\left(19\left(1-l_{2}\right)^{2}+3+15 l_{2}\right)+4 l_{1}\left(17\left(1-l_{2}\right)^{3}-38\left(1-l_{2}\right)^{2}+15\left(1-l_{2}\right)-12\right) \\
& \left.-4 l_{2}\left(17\left(1-l_{2}\right)^{2}-2\left(1-l_{2}\right)+3\right)\right) H_{3}^{2} c^{ \pm 2} q^{ \pm 2} l_{1}^{2}+\left(52 l_{1}^{3}\left(1-l_{2}\right)-12 l_{1}^{2}\left(9-8 l_{2}\right)-4 l_{1}\left(13\left(1-l_{2}\right)^{3}\right.\right. \\
& \left.\left.-19\left(1-l_{2}\right)^{2}-6\left(1-l_{2}\right)-6\right) H_{3}^{2} c^{ \pm 2} q^{ \pm} l_{1}+\left(l_{1}^{2}\left(5-8 l_{2}\right)-l_{2}^{2}\left(5-8 l_{2}\right)\right) H_{3}^{2} c^{ \pm 2}\right) \\
& \beta_{1}^{ \pm}=\frac{1}{7} 20 c^{ \pm}\left(1-4 r q^{ \pm 2} l_{1}\left(1-l_{2}\right)\left(l_{2}-l_{1}\right)\right) \times\left(\left(-360+4320 l_{1}\left(1-l_{2}\right)\left(l_{1}-l_{2}\right) r q^{ \pm 2}\right) \beta^{ \pm 2}\right. \\
& +\left(480 H_{3}^{2} c^{ \pm} r q^{ \pm 2} l_{1}\left(1-l_{2}\right)\left(l_{1}^{3}-l_{2}^{3}\right)+1440 H_{3}^{2} c^{ \pm} r q^{ \pm 2} l_{1}^{2}\left(1-l_{2}\right)\left(l_{2}^{2}-l_{2} l_{1}\right)-120 H_{3}^{2} c^{ \pm}\left(l_{1}^{2}-2 l_{2}+l_{2}^{2}\right)\right) \beta^{ \pm} \\
& +\left(\left(12 l_{1}\left(l_{1}^{5}-l_{2}^{5}\right)+60 l_{1}^{2}\left(l_{2}^{4}-l_{2} l_{1}^{3}\right)+120 l_{1}^{3}\left(l_{1} l_{2}^{2}-l_{2}^{3}\right)\right) q^{ \pm 2}+\left(14 l_{2} l_{1}^{4}-20 l_{2}^{3} l_{1}^{2}+6 l_{2}^{5}\right) q^{ \pm}\right) H_{3}^{4} c^{ \pm 2}\left(1-l_{2}\right) r \\
& +\left(-6 l_{1}^{6}+30 l_{1}^{5}+20 l_{1}^{4}\left(-3+\left(1-l_{2}\right)^{2}\right)+60 l_{1}^{3} l_{2}\left(2-l_{2}\right)-2 l_{1}^{2}\left(7\left(1-l_{2}\right)^{4}-30\left(1-l_{2}\right)^{2}+15\right)\right. \\
& \left.\left.-2 l_{1} l_{2}\left(2-l_{2}\right)\left(7\left(1-l_{2}\right)^{2}-3\right)\right) H_{3}^{4} c^{ \pm 2} q^{ \pm} l_{1}+\left(-7 l_{1}^{4}+10 l_{1}^{2} l_{2}\left(2-l_{2}\right)+l_{2}\left(2-l_{2}\right)\left(7\left(1-l_{2}\right)^{2}-3\right)\right) H_{3}^{4} c^{ \pm 2}\right) \\
& \widetilde{\alpha}^{ \pm}\left(1-l_{2}, 1-l_{1}, \frac{1}{r}\right)=\alpha^{ \pm}\left(l_{1}, l_{2}, r\right), \\
& \widetilde{\alpha}_{1}^{ \pm}\left(1-l_{2}, 1-l_{1}, \frac{1}{r}\right)=\alpha_{1}^{ \pm}\left(l_{1}, l_{2}, r\right), \\
& \widetilde{\gamma}_{1}^{ \pm}\left(1-l_{2}, 1-l_{1}, \frac{1}{r}\right)=\gamma_{1}^{ \pm}\left(l_{1}, l_{2}, r\right), \\
& \widetilde{\gamma}_{2}^{ \pm}\left(1-l_{2}, 1-l_{1}, \frac{1}{r}\right)=\gamma_{2}^{ \pm}\left(l_{1}, l_{2}, r\right)
\end{aligned}
$$

Note that the coefficients of the equations for both modes have the same appearance. All the differences are in the choice of the corresponding expressions of $c^{ \pm}, q^{ \pm}, \alpha^{ \pm}$. The coefficients at terms describing linear dispersion $\beta^{ \pm}, \widetilde{\beta}^{ \pm}$as well as the coefficients $\beta_{1}^{ \pm}$and $\widetilde{\beta}_{1}^{ \pm}$are equal for the equations for different interfaces within the same mode. 
Acknowledgements. The research was partially supported by task $5.30 .2014 / \mathrm{K}$ of the national targets of scientific research in Russia, Estonian Science Foundation (grant no. 7413), the targeted financing by the Estonian Ministry of Education and Science (grant no. SF0140007s11) and through support of the ERDF to the Centre of Excellence in Non-linear Studies (CENS) and the KESTA program.

Edited by: R. Grimshaw

Reviewed by: E. Pelinovsky and one anonymous referee

\section{References}

Apel, J. R., Ostrovsky, L. A., Stepanyants, Y. A., and Lynch, J. F.: Internal solitons in the ocean and their effect on underwater sound, J. Acoust. Soc. Am., 121, 695-722, 2007.

Bogucki, D. J. and Redekopp, L. G.: A Mechanism for sediment resuspension by internal solitary waves, Geophys. Res. Lett., 26, 1317-1320, 1999.

Camassa, R., Rusas, P.-O., Saxen, A., and Tiron, R.: Fully nonlinear periodic internal waves in a two-fluid system of finite depth, J. Fluid Mech., 652, 259-298, 2010.

Chin-Bing, S. A., Warn-Varnas, A., King, D. B., Hawkins, J., and Lamb, K. G.: Effects on acoustics caused by ocean solitons - Part B: Acoustics, Nonlinear. Anal.-Theor., 71, 2194-2204, 2009.

Choi, W. and Camassa, R.: Weakly nonlinear internal waves in a two-fluid system, J. Fluid Mech., 313, 83-103, 1996.

Choi, W. and Camassa, R.: Fully nonlinear internal waves in a twofluid system, J. Fluid Mech., 396, 1-36, 1999.

Craig, W., Guyenne, P., and Kalisch, H.: A new model for large amplitude long internal waves, C. R. Mechanique, 332, 525-530, 2004.

Engelbrecht, J. K., Fridman, V. E., and Pelinovsky, E. N.: Nonlinear Evolution Equations: Pitman Research Notes in Mathematics Series, 180, Longman, London, UK, 122 pp., 1988.

Funakoshi, M.: Long internal waves in a two-layer fluid, J. Phys. Soc. Jpn., 54, 2470-2476, 1985.

Funakoshi, M. and Oikawa, M.: Long internal waves of large amplitude in a two-layer fluid, J. Phys. Soc. Jpn., 55, 128-144, 1986.

Gear, J. A. and Grimshaw, R. H. J.: A second-order theory for solitary waves in shallow fluids, Phys. Fluids, 26, 14-29, 1983.

Giniyatullin A. R., Kurkin A. A., Kurkina O. E., and Stepanyants Y. A.: Generalised Korteweg-de Vries equation for internal waves in two-layer fluid, Fund. Appl. Hydrophys., 7, 16-28, 2014 (in Russian).

Grimshaw, R. H. J. (Ed.): Internal solitary waves, in: Environmental Stratified Flows, Kluwer, Boston, 1-28, 2001.

Grimshaw, R., Pelinovsky, E., and Talipova, T.: The modified Korteweg - de Vries equation in the theory of large - amplitude internal waves, Nonlin. Processes Geophys., 4, 237-250, doi:10.5194/npg-4-237-1997, 1997.

Grimshaw, R., Pelinovsky, E., and Talipova, T.: Solitary wave transformation in a medium with sign-variable quadratic nonlinearity and cubic nonlinearity, Physica D, 132, 40-62, 1999.

Grimshaw, R., Pelinovsky, E., and Poloukhina, O.: Higher-order Korteweg-de Vries models for internal solitary waves in a stratified shear flow with a free surface, Nonlin. Processes Geophys., 9, 221-235, doi:10.5194/npg-9-221-2002, 2002.
Grimshaw, R., Pelinovsky, E., and Talipova, T.: Modeling internal solitary waves in the coastal ocean, Surv. Geophys., 28, 273-298, 2007.

Grimshaw, R., Slunyaev, A., and Pelinovsky, E.: Generation of solitons and breathers in the extended Korteweg-de Vries equation with positive cubic nonlinearity, Chaos, 20, 013102, doi::10.1063/1.3279480, 2010.

Guyenne, P.: Large-amplitude internal solitary waves in a two-fluid model, CR Mecanique, 334, 341-346, 2006.

Helfrich, K. R. and Melville, W. K.: Long nonlinear internal waves, Annu. Rev. Fluid Mech., 38, 395-425, 2006.

Holloway, P., Pelinovsky, E., and Talipova, T.: Internal tide transformation and oceanic internal solitary waves, in: Environmental Stratified Flows, edited by: Grimshaw, R. H. J., Kluwer, Boston, 29-60, 2001.

Jackson, C. R.: An atlas of internal solitary-like waves and their properties. Prepared under contract with the Office of Naval Research Code 322PO, Contract N00014-03-C-0176, Global Ocean Associates, VA, Alexandria, VA, USA, 2004.

Jeans, D. R. G.: Solitary internal waves in the ocean: A literature review completed as part of the internal wave contribution to Morena, Rep. U-95, UCES, Marine Science Labs, University of North Wales, 1995.

Kakutani, T. and Yamasaki, N.: Solitary waves in a two-layer fluid, J. Phys. Soc. Jpn., 45, 674-679, 1978.

Knauss, J. A.: Introduction to Physical Oceanography, Prentice Hall, New Jersey, 309 pp., 1996.

Knickerbocker, C. and Newell, A.: Internal solitary waves near a turning point, Phys. Lett., 75, 326-330, 1980.

Koop, C. G. and Butler, G.: An investigation of internal solitary waves in a two-fluid system, J. Fluid Mech., 112, 225-251, 1981.

Kurkina, O. E., Pelinovsky, E., Talipova, T., and Soomere, T.: Mapping the internal wave field in the Baltic Sea in the context of sediment transport in shallow water, J. Coastal Res., Special Issue 64, 2042-2047, 2011a.

Kurkina, O. E., Kurkin, A. A., Soomere, T., Pelinovsky, E. N., and Rouvinskaya, E. A.: Higher-order (2+4) Korteweg-de Vries-like equation for interfacial waves in a symmetric three-layer fluid, Phys. Fluids, 23, 116602, doi:10.1063/1.3657816, $2011 \mathrm{~b}$.

Kurkina O., Singh N., and Stepanyants Y.: Structure of internal solitary waves in two-layer fluid at near-critical situation, Comm. Nonlin. Sci. Num. Simulation, 22, 1235-1242, 2015.

Lamb, K. G.: Conjugate flows for a three-layer fluid, Phys. Fluids, 12, 2169-2185, 2000.

Lamb, K. G.: Extreme Internal Solitary Waves in the Ocean: Theoretical Considerations, Preprint, University of Waterloo, 109117,2006

Lamb, K. G. and Yan, L.: The evolution of internal wave undular bores: comparisons of a fully nonlinear numerical model with weakly nonlinear theory, J. Phys. Oceanogr., 26, 2712-2734, 1996.

Leppäranta, M. and Myrberg, K.: Physical Oceanography of the Baltic Sea, Springer Praxis, Berlin, Heidelberg, New York, 378 pp., 2009.

Marchant, T. R. and Smyth, N. F.: Soliton interaction for the extended Korteweg-de Vries equation, IMA J. Appl. Math., 56, 157-176, 1996.

Mehta, A. P., Sutherland, B. R., and Kyba, P. J.: Interfacial gravity currents. II. Wave excitation, Phys. Fluids, 14, 3558-3569, 2002. 
Miles, J. W.: On internal solitary waves, Tellus, 31, 456-462, 1979.

Mirie, R. M. and Pennell, S. A.: Internal solitary waves in a twofluid system, Phys. Fluids A-Fluid, 1, 986-991, 1989.

Muller, P. and Briscoe, M.: Diapycnal mixing and internal waves, Oceanography, 13, 98-103, 2000.

Nakoulima, O., Zahibo, N., Pelinovsky, E., Talipova, T., Slunyaev, A., and Kurkin, A.: Analytical and numerical studies of the variable-coefficient Gardner equation, Appl. Math. Comput., 152, 449-471, 2004.

Nayfeh, A. H.: Perturbation Methods, Wiley, New York, 437 pp., 2000.

Newell, A. C.: Solitons in Mathematics and Physics, SIAM, Philadelphia, 244 pp., 1985.

Osborne, A. R.: Nonlinear Ocean Waves and the Inverse Scattering Transform, Elsevier, San Diego, 917 pp., 2010.

Osborne, A. R., Onorato, M., Serio, M., and Bergamasco, L.: Soliton creation and destruction, resonant interactions, and inelastic collisions in shallow water waves, Phys. Rev. Lett., 81, 35593562, 1998.

Ostrovsky, L. A. and Stepanyants, Y. A.: Do internal solitions exist in the ocean?, Rev. Geophys., 27, 293-310, 1989.

Ostrovsky, L. A. and Stepanyants, Y. A.: Internal solitons in laboratory experiments: comparison with theoretical models, Chaos, 15, 037111, doi:10.1063/1.2107087, 2005.

Pelinovskii, E. N., Polukhina, O. E., and Lamb, K.: Nonlinear internal waves in the ocean stratified in density and current, Oceanology, 40, 757-766, 2000.

Pelinovsky, E. N., Polukhina, O., Slunyaev, A., and Talipova, T.: Internal solitary waves, in: Solitary Waves in Fluids, edited by: Grimshaw, R. H. J., WIT Press, Southampton, Boston, 85-110, 2007.

Pullin, D. I. and Grimshaw, R. H. J.: Finite-amplitude solitary waves at the interface between two homogeneous fluids, Phys. Fluids, 31, 3550-3559, 1998.

Reeder, D. B., Ma, B. B., and Yang, Y. J.: Very large subaqueous sand dunes on the upper continental slope in the South China Sea generated by episodic, shoaling deep-water internal solitary waves, Mar. Geol., 279, 12-18, 2011.

Rusås, P.-O. and Grue, J.: Solitary waves and conjugate flows in a three-layer fluid, Eur. J. Mech. B-Fluid., 21, 185-206, 2002.

Ruvinskaya, E. A., Kurkina, O. E., and Kurkin, A. A.: A modified nonlinear evolution equation for internal gravity waves in a three-layer symmetric fluid, Transactions of the Nizhny Novgorod R. E. Alekseev State Technical University, 83, 30-39, 2010 (in Russian).

Sabinin, K. and Serebryany, A.: Intense short-period internal waves in the ocean, J. Mar. Res., 63, 227-261, 2005.
Sandstrom, H. and Elliott, J. A.: Internal tide and solitons on the Scotian Shelf: A nutrient pump at work, J. Geophys. Res., 89, C6415, doi:10.1029/JC089iC04p06415, 1984.

Soomere, T.: Coupling coefficients and kinetic equation for Rossby waves in multi-layer ocean, Nonlin. Processes Geophys., 10, 385-396, doi:10.5194/npg-10-385-2003, 2003.

Sridevi, B., Murty, V. R., Sadhuram, Y., and Murty, V. S. N.: Impact of internal waves on the acoustic field at a coastal station off Paradeep, east coast of India, Nat. Hazards, 57, 563-576, doi:10.1007/s11069-010-9567-9, 2011.

Stastna, M. and Lamb, K. G.: Sediment resuspension mechanisms associated with internal waves in coastal waters, J. Geophys. Res., 113, C10016, doi:10.1029/2007JC004711, 2008.

Stober, U. and Moum, J. N.: On the potential for automated realtime detection of nonlinear internal waves from seafloor pressure measurements, Appl. Ocean Res., 33, 275-285, 2011.

Talipova, T., Pelinovskii, E., and Grimshaw, R. H. J.: Transformation of a soliton at a point of zero nonlinearity, JETP Lett., 65, 120-125, 1997.

Talipova, T., Pelinovsky, E., Lamb, K., Grimshaw, R. H. J., and Holloway, P.: Cubic nonlinearity effects in the propagation of intense internal waves, Dokl. Earth Sci., 365, 241-244, 1999.

Vlasenko, V., Stashchuk, N., and Hutter, K.: Baroclinic Tides: Theoretical Modeling and Observational Evidence, Cambridge University Press, Cambridge, 351 pp., 2005.

Warn-Varnas, A., Chin-Bing, S. A., King, D. B., Hawkins, J., and Lamb, K. G.: Effects on acoustics caused by ocean solitons, Part A: Oceanography, Nonlinear Anal.-Theor., 71, 1807-1817, 2009.

Weisstein, E. W.: "Sawada-Kotera Equation", available at: http:// mathworld.wolfram.com/Sawada-KoteraEquation.html (last access: 19 February 2014), 2002a.

Weisstein, E. W.: "Kupershmidt Equation", available at: http: //mathworld.wolfram.com/KupershmidtEquation.html (last access: 19 February 2014), 2002b.

Yang, Y. J., Fang, Y. C., Tang, T. Y., and Ramp, S. R.: Convex and concave types of second baroclinic mode internal solitary waves, Nonlin. Processes Geophys., 17, 605-614, doi:10.5194/npg-17605-2010, 2010.

Zahibo, N., Slunyaev, A., Talipova, T., Pelinovsky, E., Kurkin, A., and Polukhina, O.: Strongly nonlinear steepening of long interfacial waves, Nonlin. Processes Geophys., 14, 247-256, doi:10.5194/npg-14-247-2007, 2007.

Zwillinger, D.: Handbook of Differential Equations, 3rd editon, Academic Press, Boston, USA, 834 pp., 1997. 